\title{
From Documents to Data: A Framework for Total Corpus Quality
}

Miriam Hurtado Bodell1,a, Måns Magnusson² and Sophie Mützel

1) Linköping University

2) Uppsala University

3) University of Lucerne

a) Corresponding author: miriam.hurtado.bodell@liu.se

\section{Introduction}

The combination of available large-scale digitized corpora as well as novel tools from fields such as natural language processing (NLP) and machine learning have elevated computational text analysis in empirical studies of the social world (e.g. see Edelmann et al. 2020; Evans and Aceves 2016; Grimmer, Roberts, and Stewart 2021; Molina and Garip 2019; Wilkerson and Casas 2017 for overviews).

Computational tools increasingly grant social scientists insight into numerous social phenomena, events, situations, and relations through analysis of vast amounts of digitized text data (DiMaggio, Nag, and Blei 2013; Fligstein, Brundage, and Schultz 2017; Fuhse et al. 2020; Gentzkow, Kelly, and Taddy 2019; Grimmer 2010; Grimmer and Stewart 2013; Hoffman et al. 2018; Mützel 2015; Rule, Cointet, and Bearman 2015). As the quantity of digital and digitized text data sets continues increasing globally, approaches to gauging and diagnosing the quality of processed machine-readable corpora grow increasingly crucial. Understanding error structures and, conversely, the validity and reliability of largescale computational text analyses, remain-despite their importance for social scientific inquirieslargely underdeveloped (see Hsieh and Murphy 2017; Olteanu et al. 2019; Sen et al. 2019).

Collections of documents (henceforth corpora) have long furnished matter for social inquiries into both the present and the past, proliferating an understandably profound diversity of research approaches. Some utilize textual materials qualitatively: as sources from which to gain insights into and to describe 
particular phenomena, focusing on explicit content (e.g. Bail 2016b; Franzosi 1989), seeking to understand their particularities, or, in the hermeneutic tradition, using texts as sources for interpretation and investigations into the meanings of events (e.g. Wagner-Pacifici 2010). Others use texts as "social sensors," to relate textual inferences to phenomena outside the texts themselves (Hoffman et al. 2018; Mohr 1998; Rule et al. 2015). Still others treat texts purely as data, working quantitatively to produce replicable generalizations (Agiatis et al. 2017).

All social scientists working with processed machine-readable corpora (i.e. the best possible digital representations of original content which computers can read), before seeking scientific inferences, must address a fundamental question: Is my corpus of sufficient quality to answer my research question? In this paper, we propose a total corpus quality framework for assessing textual data as a cohesive conceptual apparatus enabling researchers to diagnose an individual corpus' scientific value along three dimensions: total corpus error, corpus comparability, and corpus reproducibility. By mapping three key components of total corpus error, we can answer the questions: how much of the original content is still available in the source materials? (source errors), how different is the processed machine-readable corpus from the original corpus? (textual representation errors), and what is the discrepancy between the inference we aim to make and what we have measured? (research inference errors). This first dimension-total corpus error-allows us to reason about how near the textual material at hand allows us to approach our particular research question's "true" answer. The second dimension-corpus comparability-indicates how well estimates and variables from the available corpus compare both with subsets of the given corpus and to related estimates from other corpora. The third and last dimensioncorpus reproducibility-highlights the advantages of transparency and openness in the data curation ${ }^{1}$ process.

The concept of quality in evaluating data is hardly unique to the social sciences. Over the vast literature on survey methodology, the concept of data quality rests on the notion of "fitness of use." This 
straightforward definition serves as a standard for data quality across multiple dimensions and highlights different error sources relevant to the data's use in scientific inquiry (Biemer and Lyberg 2003; Juran and De Feo 2010; Pipino, Lee, and Wang 2002). ${ }^{2}$ The total survey error framework commonly helps in understanding the accumulation of all errors possibly arising from the design, collection, processing, and analysis of survey data (Biemer 2010). Analogously, and closely related to notions developed in survey methodology, we develop a framework to assess textual data quality. The difference in analyzing textual data rather than survey data is important. The authors of many corpora, unlike subjects in surveys or interviews, never imagine their texts' involvement in research. Hence, the contents of most corpora need not relate, a priori, to investigations by researchers (who concordantly exercise only limited control over the production process and can know little a priori about data quality). Further, the steps required to collect and curate textual data most often differ from those for survey data, yielding a distinct error structure. Approaching the quality of a textual corpus from a perspective of "fitness of use" directs our attention to crucial aspects of curating and preparing data for scientific inference before running analyses. By carefully exploring quality dimensions and potential sources of error, researchers can diagnose the scientific value of each corpus and the knowledge they may gain from it. Such an evaluation, in turn, allows reflection on different error sources' potential impacts on scientific conclusions; it also enables collaborative data curation.

For one, this paper contributes to discussions of the importance of determining the nature of errors in a given body of textual data to understand their potential effects on scientific inquiry (Franzosi 2004). It also contributes to a growing literature on the consequential decisions taken in curation and preprocessing prior to analysis of corpora (Barberá et al. 2021; Björk 2015; Bode 2020; Denny and Spirling 2018; Geiger et al. 2020; Jo and Gebru 2020; Nguyen et al. 2020; Schofield, Magnusson, and Mimno 2017; Sen et al. 2019; Tahmasebi and Hengchen 2019). Moreover, it contributes to ongoing discussions bridging the oft-proclaimed chasm between qualitative and quantitative methods (Olsen 2004). Both 
approaches use the same data sources, face similar problems, and follow similar inductive and deductive strategies to reach scientific conclusions (Baumer et al. 2017). We hold that both approaches also face similar-but not identical-issues in data curation. Additionally, we tie together insights from the long tradition of understanding documentary sources (Fickers 2012; Scott 1990) in terms of the challenges that arise when the sizes of corpora increase. The paper also contributes to the advancement of research practices. Typically, in the social sciences, only a few possible corpora may aid addressing any particular research question. Even when alternative corpora are available, getting acquainted with such other corpora tends to be highly resource-consuming. Thus, diagnosing and evaluating the strengths and weaknesses of a textual corpus before starting computational analyses is crucial for most researchers.

To the best of our knowledge, no cohesive scientific framework for evaluating the quality of corpora currently enables identifying distinct error sources, measuring their magnitudes, and discussion of their bearing upon scientific inference. Previous work focuses on developing frameworks for specific data sources (e.g. Twitter, Hsieh and Murphy 2017; Sen et al. 2019), or specific data types (e.g. event data, Demarest and Langer 2019) or non-textual "big data" (Amaya, Biemer, and Kinyon 2020). By contrast, in this paper, we develop a framework for approaching textual data quality from a general perspective to categorize errors potentially affecting scientific inference. For illustrative purposes, we apply our framework to exemplary digitized archival textual data. Also unlike prior research, the total corpus quality framework is agnostic as regards the original format, methodological approaches, or corpus size.

The structure of this paper is as follows. First we develop the total corpus quality framework. We begin by suggesting that the processes of corpus creation and of scientific inquiry interrelate. We then introduce approaches to evaluating systematically the quality of textual data along three dimensions: total corpus error, corpus comparability, and corpus reproducibility. After presenting the framework, we discuss how researchers may identify and measure errors in a corpus via an example case study. The case study, using Swedish newspaper texts to examine the salience of immigration discourse, shows how 
to use the proposed total corpus quality framework to evaluate a corpus and how to draw inferences from it. We choose to evaluate the different dimensions of total corpus quality using this particular case study because the corpora creation processes include both challenges common with data originating on physical paper and issues related to inferences drawn using large-scale and/or historical data. As such, the case study exemplifies considerations and decisions fundamental to the initial stages of working with any corpus prior to computational textual analyses and illustrates strategies for diagnosing and evaluating the different dimensions of quality given normal research constraints and limitations.

\section{Total Corpus Quality}

In empirical research, texts serve either in their manifest, face-value form or as sensors reflecting social phenomena, such as what a group of people was experiencing at a specific time, or how people interpret and evaluate events, actors, or relations. Regardless of the researcher's approach, working with textual data is non-trivial. Decisions made in initial steps of data curation may impact later analyses significantly.

We suggest that empirical studies utilizing textual data entail two key elements. Figure 1 schematically presents corpus creation and scientific inquiry as two interrelated processes necessitating diagnosis and evaluation of the quality of a textual corpus.

[Figure 1. about here]

The initial steps involve first processing and curating source materials, such as historical documents, radio broadcasts, or digital data, to assemble research corpora. This transformation from original documents and transcripts to machine-readable text data occurs in several steps along the digitization process. Some sources contain additional non-textual information, such as speaker pauses and tonality, or font types and font size, while others do not. Some sources are handwritten and others printed. Nevertheless, the textual information from paper documents, audio recordings, "born-digital" text segments, or "digital trace" data (e.g. social media posts, Bail 2012; Garcia and Rimé 2019) are prone to the same kinds of errors, affecting the scientific value of the material. 
Second, researchers seek to relate their data, selected and curated, to the scientific inquiry at hand. Advances in NLP and new computational text analytic models have enabled social science researchers to gain insights into both manifest and latent phenomena of interest (Bail 2016a; Flores 2017; Rule et al. 2015). Using computational and statistical methods implies an operationalization of often-abstract research questions into concepts studied empirically using available corpora. Many such operationalizations require that researchers infer the semantic content of interest via model representations (e.g. using word embeddings or topics (Garg et al. 2017; Hurtado Bodell, Arvidsson, and Magnusson 2019; Kozlowski, Taddy, and Evans 2019) .

To formalize the discussion and the concepts of interest, we have divided the overall quality of corpora used for scientific inquiry into three aspects that together capture the total quality of the corpus for its intended research purpose, namely: (1) total corpus error, (2) corpus comparability, and (3) corpus reproducibility (see Figure 2).

[Figure 2. about here]

\section{Total Corpus Error}

We view the total corpus error as the difference between a "true" answer to the research question of interest and the actual conclusions based on the textual material at hand. A "true" answer to a research question is, realistically, merely an abstraction and unreachable in most situations, but it helps to illustrate the different sources of errors in using textual material for scientific inference.

We suggest that the difference between a concept's apparent value based upon textual data and its "true value" stems from three primary error sources: source errors, textual representation errors, and research inference errors. The first and second relate to the corpus creation processes and are measurable for most corpora. The main difference between the two is that researchers oftentimes cannot reduce source errors, as they are inherent to the original material. The textual representation 
error, on the other hand, is, at least theoretically, reducible. Research inference errors connected to the processes of scientific inquiry, as illustrated below, are generally more difficult to quantify.

\section{Source Errors}

Textual data come from various sources, including paper documents, digital text files, and audio recordings. We define source errors (SE) as the errors inherent in the physical source material, which distort the original information, making it harder for researchers to read or listen to the text. Source errors thus impact the potential quality of the corpus even prior to corpus creation. This also implies an inherent connection between source errors and the original (source) material. The source errors' structures mean they are often random in nature (e.g. a damaged page, a garbled recording). More systematic excisions or distortions of the original text we consider coverage errors (read more in Research Inference Errors section).

We can use the size of the source error to answer the question: how much of the original content is still available in the source material? For example, background noises or screams in an audio file may make a speech inaudible to any person listening to the recording. Similarly, in handwritten texts, liquids may have dissolved the ink and made the content unreadable. In "born-digital" texts, source errors are usually less problematic but may arise (e.g. if the original authors encoded the text incorrectly, making the words difficult to read). A high source error indicates that the data used in subsequent analyses will be very unlike the original source material. ${ }^{3}$ Hence, source errors weaken the signal in the corpus, limiting the potential scientific conclusions one may draw from the material, much like measurement error in the total survey quality framework.

\section{Textual Representation Errors}

Textual representation errors (TRE) capture the differences between source material and machinereadable textual representations of the material. Hence TRE answers the question: How different are the 
processed machine-readable corpus and the original corpus? A high textual representation error indicates that the machine-readable corpus is very dissimilar from the original material. ${ }^{4}$ The sizes of these dissimilarities tend to be measurable since there exists a "ground-truth" to which one may compare the digitized material straightforwardly, namely what a researcher would see studying the original material. Large dissimilarities between the original and the machine-readable material weaken the scientific value of the corpus. TRE, thus, also relates to the measurement error in the total survey error tradition, but with the difference one may estimate it by comparing the observed source material and the machine-readable representation.

Textual representation errors may occur at several steps in the corpus creation process (see Figure 1). These steps include creating a first machine-readable representation of the data, turning the textual content into documents, and enriching the corpus before finalizing the material. We therefore categorize the textual representation errors into four main categories: source-to-(digital)-text errors, text-todocuments errors, documents-to-corpus errors, and processing errors.

Source-to-(Digital)-Text Errors. Researchers may perform the processes of going from observed sources-be they printed texts or audio recordings - to machine-readable representations either manually or using more-or-less-automatized computational methods. ${ }^{5}$ Researchers are generally able to read documents, to interpret additional information encoded in the document structure (e.g. title and image captions), and to write it down in a machine-readable form. While manual (human) transcription is slow and requires extensive work when handling many documents, computers offer vast scaling opportunities but struggle to interpret natural language or visual cues as human researchers can. Further, errors produced by humans tend to be random, at least when numerous annotators transcribe texts within the same corpus. Computers, on the other hand, tend to produce systematic errors. Creating a machine-readable corpus via manual transcription typically requires only one step, while automated transcription entails multiple steps. We thus divide source-to-(digital)-text errors into two components: 
source-to-digital errors and digital-to-text errors. The second occurs when the transcription process employs computational means.

In the case of manually creating a machine-readable representation of documents or audio, the error structure is similar to that of coding errors in the total survey quality framework. Individual transcribers might introduce systematic errors, but, when using multiple transcribers, with documents allocated randomly, the error structure is random rather than systematic.

Source-to-Digital Errors. Whenever converting an analog document into a machine-readable representation, one must depict the physical pages as images. Similarly, one must convert analog audio, such as vinyl records, cassettes, and CDs, analogously into digital audio representations. Source-to-digital errors consist of artifacts (i.e. any differences between the source text and that in the corresponding digital rendering) introduced in the processes of (1) generating a digital image of original analog text or (2) generating an audio file from an original analog recording. Examples of source-to-digital errors are dust caught between documents and scanners or creases, folds, or discolorations of source pages caught or enhanced in the digitized images (Conway 2011; Terras 2011), difficulties in handling while scanning (e.g. books Read 2000), or interference or humming when digitizing audio (Iraci, Hess, and Flak 2020). Source-to-digital errors differ from source errors since they occur during the corpus creation process. That is, an incorrigible ink-spill in the source materials is a source error, but an unintentional folding of a document during scanning is a source-to-digital error.

Digital-to-Text Errors. When utilizing computers to transform analog texts or audio into digital text, one step from the original format to a machine-readable text segment is typically necessary. The initial digital representation will be either a scanned image or a digital audio recording. Additional errors may occur in the process of extracting the textual content: We call these digital-to-text errors. Conceptually, 
digital-to-text errors come in two types, one specific to transforming images to text, and one to transforming audio to text.

Image-to-text errors capture how often the textual unit of interest differs between digital images and machine-readable texts. Image-to-text errors are problematic in that they may create nonsensical or "faux content," (e.g. additional characters, potentially altered words or the text passages' meanings (Hill and Hengchen 2019; van Strien et al. 2020). Converting images of texts to machine-readable text may systematically misidentify (and thus transform) some characters (or words), due, for example, to visual similarities (e.g. between the letters "c" and "e") (Jarlbrink and Snickars 2017; Tanner, Muñoz, and Ros 2009). Image-to-text errors may also occur given misinterpretation of some textual content as nontextual content (e.g. categorizing newspaper headlines as images rather than texts due to their large font). Image-to-text errors entail misinterpretations of characters by algorithms rather than distortions of the original images, distinguishing them from source errors.

Just as one must translate images into machine-readable text before using them in computer-assisted analysis, one must also translate audio into machine-readable text, either by hand or using computational methods (e.g. Baevski et al. 2020). Hence, audio-to-text errors describe how often the textual unit of interest differs between the original audio and the machine-readable textual content. Audio-to-text errors, unlike image-to-text errors, may occur due to homophones (e.g. "allowed" vs. "aloud"), which one can only infer from context. Hence, the conceptual difference between image-totext and audio-to-text errors is small.

Text-to-Documents Errors. Textual documents consist of cohesive units of text. Deciding the most appropriate text unit is not a straightforward task. Some researchers may see paragraphs as natural units (Algee-Hewitt et al. 2015), while other researchers may find news articles or sections thereof better units (DiMaggio et al. 2013). Similarly, we must define relevant segments within audio recordings to create 
cohesive text units, such as from radio segments. For large data, automatic segmentation procedures find cohesive text or audio units, commonly either splitting or combining parts of an image or audio recording into cohesive text segments (Shinde and Chougule 2012; Smith 2007; Zhan, Wang, and Gao 2006). We refer to errors related to creating coherent text segments, therefore, as text-to-document errors. These errors occur when segmentation procedures split cohesive texts incorrectly, creating units of analysis different from those researchers would select. Errors in creating coherent text segments may occur through (1) incorrectly combining content from different documents into one cohesive segment, or (2) splitting the content of one document into multiple incorrect segments. For example, one may (1) combine multiple news articles on one page into a single article or (2) split a news article spanning multiple pages into multiple segments, creating "faux documents." The severity of the problems associated with the different possible text-to-documents errors hinges on the relevant research question and how one operationalizes the research questions.

Documents-to-Corpus Errors. The third type of error source for TRE occurs while enriching textual data with further information, which we refer to as meta-data (information about a text's context, authors, or origins not part of the actual textual content). Documents-to-corpus errors describe the accuracy of meta-data in a corpus. Consider a corpus of fake news items containing meta-data representing the origin of the speech (Wang 2017), or a corpus of blog posts possibly holding labels signifying political leaning (Roberts, Stewart, and Tingley 2019). In these cases, the documents-to-corpus error represents how often the labels for origin or political leaning are incorrect.

Processing Errors. Any data-processing procedures toward obtaining a final processed machinereadable version of the corpus may inadvertently introduce additional discrepancies between the original documents and the data. Processing errors are additional discrepancies introduced through the process of going from the initial digital representation of the corpus to a machine-readable version. Processing errors can occur, for example, when markup languages, such as HTML, appear in the 
machine-readable text: errors introduced by bugs in the code, or introduced when translating a corpus across different digital schema (Mesiti et al. 2006; Mitchell 2018).

\section{Research Inference Errors}

The errors introduced thus far have mostly been technical and usually mitigatable through technical means. In contrast, research inference errors (RIE) relate closely to the process of scientific inquiry, distinguishing RIE from TRE. RIE are therefore consequential in empirical research and may be substantial even in a small-scale study. RIE answers, based on the corpus and on the decisions made in corpus curation, the question: what is the discrepancy between the inference we aim to make and our measurement? These errors capture the textual data's "fitness of use" relative to the research question at hand. Compared to TRE, most RIE are less straightforward to measure since knowing the correct "true" answer to a research question is typically impossible. ${ }^{6}$

Research inference errors are further divisible into four types: coverage errors, sampling errors, text curation errors, and specification errors.

Coverage Errors. Coverage errors are the discrepancies between the inference target population, as defined by the research question, and the texts available in the corpus. In the case of textual data, defining the target population can be more difficult than in the classical total survey framework. The target population may be the entire universe of texts, the actors who wrote the texts, or the social contexts of the texts' productions. Coverage errors can arise from lacking access to all relevant documents, those documents' non-existence, or the lack of meta-data. Coverage errors can, thereby, lead to problems of representativeness (Fickers 2012; Mogalakwe 2009; Scott 1990). The coverage error relates to both the frame error and the non-response error in the total survey error framework (Biemer and Lyberg 2003; Lyberg 2012). ${ }^{7}$ 
Depending on the source of the corpus, coverage errors may or may not rectifiable. If data for integral parts of a social world are non-existent, this may lead to a sizeable coverage error because essential dimensions of the cultural world are unavailable for analysis (Dobson and Ziemann 2020; Mohr et al. 2020; Pechenick, Danforth, and Dodds 2015). For example, surviving historical records in archives and libraries typically concern the wealthy, the educated, and the victors of war-unrepresentive of most of the populations and thus possibly hindering insights into the general target population (Bode 2020; Harris 2002; Mohr et al. 2020). This error hence also relates to the concept of source criticism, and its call to consider the intent behind and the authenticity of historical documents (Dobson and Ziemann 2020).

Lack of coverage is also a prominent feature of contemporary digital trace data, problematizing inferences about general populations (Bruns and Highfield 2013; Mellon and Prosser 2017; Ruths and Pfeffer 2014). The well-known problems of differing degrees of accessibility of social media platform data (Boyd and Crawford 2012; Giglietto, Rossi, and Bennato 2012) and missing sociodemographic metadata in digital trace data also remain concerns for large corpora (Salganik 2017).

Sampling Errors. The introduction of sampling errors occurs following subsampling from a full corpus to draw conclusions outside the observed sample. Researchers may employ sampling to reduce the number of documents in their analysis, e.g. for computational reasons or for more complex manual close reading. The sampling error equals the discrepancies between the information available in the full corpus and the sampled subset with respect to the target population and to the research question. Trivially, the sampling error is zero if the analysis includes the entire corpus. ${ }^{8}$ Scholars interested in using "small data," i.e. content created by single authors or small groups (Manovich 2016), may avoid sampling altogether if their target population is small enough. It can be easy to control the sampling error since the impact depends on the methodology used for sampling. It is possible to produce unbiased estimates using random sampling, but at the cost of variance (see Cochran 1977; Lohr 2009 for details). In this way, we can also reduce the total sampling error by increasing the size of the subsample. However, when 
using non-random sampling (e.g. using meta-data variables to find an appropriate data subset), the sampling error may be systematic and can introduce bias.

Text Curation Errors. Multiple researchers often use the same corpora. Guided by different research questions, they may, however, make differing decisions in their data curation. We define corpus curation as all activities changing a processed machine-readable corpus in order to answer the research question. Varied reasons may motivate curation differences, such as computational reasons (Schofield et al. 2017), reducing the vocabulary of the corpus (Schofield and Mimno 2016), or editing the corpus to reduce the noise in the analysis. Text curation errors, in turn, refer to errors introduced curating the corpus to answer a specific research question. These errors may occur unintentionally while processing and analyzing the data, or intentionally-e.g. removing documents with few words or removing stop words (Denny and Spirling 2018; Schofield et al. 2017).

Any decisions made to alter the corpus may introduce errors. If no data curation occurs, the text curation error is zero, but this might augment other error sources such as the text-to-document error, coverage error, or specification errors (see below) (e.g. if small documents removed in the data curation process contain information different from that in larger documents).

Specification Errors. Research using textual data requires the operationalization of research questions into more-or-less measurable entities. A simple example is using the ratio of occurrences of the words 'man' and 'woman' to study gender representation; another is using a sentiment lexicon to study valence. The move from abstract and theoretical concepts and questions to concrete operationalizations is central to studying social phenomena via textual data. The differences between how to measure relevant concepts and the ideal abstraction of the concept defined by the research question are specification errors, in analogy to the specification error in the total survey error framework (Biemer and Lyberg 2003). The operationalization of the research question impacts the corpus' "fitness of use." Sometimes researchers need to consider several operationalizations, for example, in a study of the 
salience and framing of abortion in political campaign speeches. Such a study would have to: (1) measure whether a speech, or part of a speech, addresses abortion in a sense relevant to the research question, and (2) decide how to measure framing. Different operationalizations of (1) and (2) would introduce different errors of differing magnitudes and structures with respect to the conclusions drawn from the data.

\section{Bias and Variance}

The total corpus error captures all the different error sources that affect the scientific conclusions drawn from texts. Analogous to the total survey error (Biemer and Lyberg 2003; Lyberg 2012), we can further elaborate how total corpus error affects the scientific results by separating the total error into bias and/or variance. Let $\theta$ be the "true" value of interest and $\hat{\theta}(\boldsymbol{w})$ an estimator of that true value as regards a corpus (or data) $\boldsymbol{w}$. We further assume our corpus comes from a "true" data-generating process $p_{\text {true(w) }}$. We may then separate the total (mean squared) error of our estimator as

$$
E_{p_{\text {true }(\mathrm{w})}}(\hat{\theta}(\boldsymbol{w})-\theta)^{2}=\operatorname{Bias}(\hat{\theta}(\boldsymbol{w}))^{2}+\operatorname{Var}(\hat{\theta}(\boldsymbol{w}))
$$

All the errors presented will affect the total (mean squared) error of our estimator, but the effect on the scientific inference depends on whether the error will affect the bias or the variance of the estimator. Bias relates closely to validity, in that an increased bias will reduce the validity of the conclusions (i.e. the extent to which the results $(\hat{\theta}(\boldsymbol{w}))$ measure what they are supposed to measure $(\theta)$ over many different data realizations). On the other hand, variance relates closely to reliability and affects the extent to which results are reproducible and which conclusions one may draw from smaller corpora (Golafshani 2003). Hence, understanding the different sources of errors and the transparency of potential error sources is important to avoiding incorrect conclusions from both large and small corpora (Nelson 2019). In general, tasks performed by humans - such as manual annotation - tend toward both high variance and high bias for each individual, although, in the final material, we can reduce bias from individual 
annotators by allocating multiple persons randomly to a single task, such as transcribing textual data. Tasks performed by machines - such as automatic annotation - tend on the other hand toward high bias and low variance. We can represent each study using a given corpus as a dart thrown at the dartboard, where the bullseye signifies the true value of the concept of interest $(\theta)$. We may take each dart throw at the dartboard to represent one study using a given corpus coming from $p_{\text {true(w) }}$, resulting in an estimate $\theta$. An estimator $\hat{\theta}(\boldsymbol{w})$ with low variance and high bias indicates that repeated studies using new data should be similar to one another but not necessarily near the true value (the bullseye, $\theta$ ). Instead, $\hat{\theta}(\boldsymbol{w})$ with large variance and small bias would yield varying estimates of the same concept, but all clustered around the true value (see Figure 3). Hence, adding more data may reduce variance but not bias.

\section{[Figure 3. about here]}

Consider an over-simplified example in which we are interested in the proportion $p$ of the words 'man' and 'woman' in a corpus as a simple study of the representation of gender. Assume further that a random mechanism has substituted one for the other (and vice verse) in documents in the corpus. Within a single document or a small corpus, additional random noise may affect conclusions regarding the use of the words. Adding more documents, the effects of random noise (variance) on our findings would, however, decrease. Then the (small) bias introduced by the random mechanism will become the main problem. However, if only 'man' randomly becomes 'woman,' the bias would be much larger, and adding more data would help us less in estimating $p$ from our corpus or in reducing the total error. This simple example highlights the fact bias remains a problem even as larger and larger data repositories become available to social science researchers, because adding more data cannot mitigate them. Table 1 summarizes the different components of the total corpus error and its impact either on the bias or variance of the estimates of interest.

[Table 1. about here] 


\section{Corpus Comparability}

The second dimension of the total corpus quality framework is corpus comparability, the degree to which an extracted estimate is comparable to other estimates of the same concept, both within the same corpus and between other corpora of relevance. We consider corpus comparability to have two components: within-corpus comparability and between-corpus comparability.

\section{Within-corpus comparability}

High within-corpus comparability guarantees that an estimate of a concept, such as gender representation or the salience of a political topic in newspapers, is comparable across the entire corpus. This does not require an estimate stable over all different subgroups (e.g. the salience of a football topic will likely be higher in a newspaper's sports section). Instead, high within-corpus comparability means that the error structures are stable and correlate with other meta-data of interest. One may hence estimate within-corpus comparability by studying the total corpus error in different parts of the corpus relevant to the research question (e.g. different periods or political parties in parliament). This definition of high within-corpus comparability follows the common notion that having the same constant error throughout the whole corpus is preferable. Issues with within-corpus comparability may, for example, arise in cases using different digitization technologies for separate parts of the corpus. Such practices can result in varying biases and variances in the estimates for the distinct groups, making comparisons difficult and possibly precipitating spurious conclusions.

\section{Between-corpus comparability.}

Many research questions require enriching or combining information from different sources or comparing the findings for different corpora. Between-corpus comparability expresses the degree to which estimates from the corpus of interest are useful for cross-corpus comparisons. Not unlike withincorpus comparability, between-corpus comparability measures similarity of error structures between two or more corpora. Low between-corpus comparability may limit the possibility of comparing results with previous literature and theory or enriching the corpus with external data sources. 


\section{Corpus Reproducibility}

Our framework considers corpus reproducibility as the third quality dimension of a corpus. Here we apply the term 'reproducibility' to the corpus: two different researchers should be able to create the same corpus from the same source material. Readers may be more accustomed to the idea of findings' reproducibility, i.e. the potential one researcher could take the original data and the code used for analysis in some prior study and reproduce all the numerical findings (Camerer et al. 2016; Liu and Salganik 2019; Open Science Collaboration 2015). ${ }^{9}$ However, data itself should also be reproducible (Downing 2004). If the methodologies used when the sources to a processed machine-readable corpus are deterministic, then re-using the same methodology would recreate the same corpus. However, if redepicting documents and converting them into processed machine-readable text require humanly annotated materials, corpus reproducibility may be more problematic. Therefore, we view the potential of reproducing a corpus as highly dependent on the sources and the magnitude of the source and textual representation errors.

One may, at least in theory, evaluate corpus reproducibility by re-starting the processes of corpus creation (i.e. re-tracing the original documents to processed machine-readable data selected for a specific research question and studying how much of the processed machine-readable corpus remains consistent). We may thus assess corpus reproducibility by comparing the textual representation error and its elements for two corpora created by researchers who began with the same set of original documents. If all error components are roughly the same size, we can see the corpus as reproducible. Furthermore, measurements of inter-coder reliability (Bais et al. 2019; Belur et al. 2021; Campbell et al. 2013) may reflect how much randomness exists in the corpus creation procedures (e.g. when enriching the corpus with new meta-data or using manual annotations). 
Low corpus reproducibility affects the variance of the estimates of interest-due to differences in data curation, repeated studies will likely yield different results. Documentation and publicly accessible code of all relevant decisions made in the data collection and data curation processes help to promote high corpus reproducibility.

Minimizing the total error and maximizing total corpus quality Listing the different error structures may lead us to believe that the goal for the researcher is to minimize all error sources. Two considerations may make this difficult.

First, in most situations, researchers work under budget constraints. Creating an error-free corpus is simply unaffordable, and so we must instead balance the costs of reducing different errors to minimize the total error given the resources at hand. As an example, given unlimited resources multiple researchers could extract and manually check all textual content and meta-data with high domain knowledge, creating an error-free processed machine-readable corpus. As sampling loses its necessity when computational resources are endless, research inference errors also shrink. This is, clearly, only a researcher's dream; In practice, researchers must make decisions in order to maximize total corpus quality given limited resources.

Second, there are trade-offs. We can improve total corpus quality in one aspect while, at the same time, reducing it in another. One example is focusing on reducing the TRE for a particular subgroup, e.g. all audio from podcasts on the top charts, reducing the total error but reducing with it the within-corpus or between-corpus comparability. However, solely conforming to existing standards or definitions may not always be the best alternative. Breaking from traditional methods to use new measurement techniques may reduce comparability and yet decreasing the specification error may render it defensible. 
Hence, this framework will not solve the problems challenging researchers using corpora for research but will aid discussing the trade-offs that inevitably guide the curation of text data and the design of textanalytic studies.

\section{Total Corpus Quality in Practice: From Document to Data to Knowledge}

The processes of transforming original sources into high-quality data usable for research depend on the particular research questions, original materials, and resources at hand. Here we provide a case study to demonstrate how one might use the framework. The Case Study: Curating the Swedish National Newspaper Corpus 1945-2019 for Studying Salience of Immigration Discourse

Our case study draws on a digitized newspaper archive spanning seventy-five years of news coverage of Sweden's modern immigration history. Substantively, it explores when and how much the national news in Swedish national media has discussed immigration? We operationalize Swedish news media with Sweden's four national newspapers-Aftonbladet, Dagens Nyheter, Expressen, and Svenska Dagbladetduring 1945-2019. This corpus includes more than 100,000 unique newspaper editions. Visible in the examples in Figure 4, over those seventy-five years newspapers have shifted dramatically in appearance, from homogeneous font styles and tightly packed textual content to pages filled with infographics and a variety of font styles and sizes.

[Figure 4. about here]

Like many research enterprises, we are dependent on data digitized by a third party, in our case by the National Library of Sweden (NLS). NLS has digitized its archive to store digital versions of fragile original materials, using both automatic segmentation and optical character recognition (OCR). ${ }^{10}$ Given NLS's interest in digitizing content, they created the digitized newspaper data without considering later scientific inquiries. For example, the OCR procedure extracted from the original newspaper material 
textual contents stored in text blocks, which may, but do not necessarily, map to humanly recognizable units such as paragraph or article (see Figure 5 for an example).

[Figure 5. about here]

We want to enrich the corpus, using meta-data to indicate whether the textual content is editorial or commercial, body text or not, and to label section identifiers. Section identifiers are recurrent titles, logos, or images identifying similar section content over time. Enriching the corpus with such meta-data enables us to filter systematically the vast materials of the Swedish National Newspaper Corpus 19452019 and, in turn, to select specific parts of relevance to our research question.

\section{Total Corpus Error}

We can separate the total corpus error into two main steps, the textual representation error and the research inference error.

Step 1: Measuring Textual Representation Error. We first create a "ground-truth" to which to compare the processed machine-readable version of the corpus. We use human annotators to create this "gold standard," i.e. the text a human would read, which we can use to compare the information in the original and the machine-readable data, respectively. One benefit of such a gold standard is its reusability following new digitization efforts of the same corpus or, for example, improved OCR processes.

Creating a gold standard is costly, however, and so we evaluate the errors instead by using random sampling to obtain a representative sample of the full corpus. This strategy enables unbiased estimates of the total errors in a cost-effective way (Cochran 1977; Lohr 2019). We are interested in comparisons between different newspapers and periods, and therefore utilize a stratified random sampling procedure to ensure that these features are represented in the selected sample (see Cochran 1977 and Lohr 2009 for details). ${ }^{11}$ In our case study, a sample of 120 pages suffices to evaluate the size of four possible error 
sources (i.e. source-to-digital error, digital-to-text error, text-to-document error, and processing error). We lack access to the source material (i.e. the physical copies of the newspapers), and rely therefore on images of the material. It is therefore not possible for us simply to evaluate the source error separately.

First, a human annotator takes note of each visible artifact distorting the original analog edition of the page in the sample. The number of visual artifacts is our estimate of the combined source error and source-to-digital error. Second, to measure the digital-to-text error-more specifically, the image-to-text error-the annotator manually writes down the text, ignoring font differences while respecting upperand lower-casing. ${ }^{12}$ Third, the annotator assigns all text blocks on the sampled pages to a given article. These connections between text blocks and articles become the ground truth, or gold standard, from which we measure text-to-documents error, i.e. how often the segmentation reflects the news articles' boundaries. Fourth, we investigate the processing errors by comparing the content of the first digital version of the text-available via an API at the SNL - with the final stored version.

To enrich the corpus with meta-data regarding the type of content in our text blocks, we approach this as a machine learning problem, much like Barberá et al. (2021). To train our classification models, we need training and test data (Murphy 2012). We use the training set to create the classification model and the test data to evaluate it, seeking to avoid overfitting (Hawkins 2004), since we want to find a model generalizable to the rest of the corpus. We use around 50,000 text blocks as our training set and 10,000 text blocks as the test set in creating our meta-data. In total, our material makes up a total of 720 different newspaper pages. ${ }^{13}$ We train and evaluate three separate classification tasks, one per variable of interest: whether the textual content of a text block is editorial (Task 1), if a text block contains editorial body text (Task 2), and if a text block contains a section title (Task 3). We choose these tasks because we are interested in Swedish immigration discourse and believe that editorial body texts in national news sections will best capture this. We let two annotators annotate this material since we anticipate especial difficulty in the meta-data enrichment task. The annotation for creating meta-data 
labels takes approximately three weeks of full-time work. We also let both annotators label parts of the same material, ${ }^{14}$ allowing us to calculate measures of inter-coder reliability. For the final training and test data, we use the label of the more senior annotator.

Step 2: Estimation and Analysis of Errors. Our second step is to measure the textual representation error by comparing the layout, textual content, and meta-data obtained via manual annotation to the processed machine-readable corpus.

Source-to-Digital Errors. In our case study, the annotator does not find any artifacts of the depiction of the newspaper pages in the sample. However, we have found some instances of source-to-digital error (see example in Figure 6) during further work with the material. Hence, our estimate of the sourceto-digital error is small. For our purposes, the source error and the source-to-digital error in our corpus are hence negligible.

[Figure 6. about here]

Digital-to-text errors. We investigate the image-to-text error using two different measurements: (1) Levenshtein distance and (2) word recognition. Levenshtein distance calculates the number of edits (deletions, insertions, or substitutions) needed to transform one string into the other. We use the Levenshtein distance to compare the digitized and annotated textual content from each sampled paragraph (Navarro 2001). This estimate captures character-level accuracy. Second, we study word recognition, i.e. how many of the words in the digitized texts exist in the annotated material (regardless of position). This captures word-level accuracy. Lastly, we compare the image-to-text error for both the raw material and the material after some preprocessing-i.e. removing all alphanumerical characters and excessive white spaces - to get more in-depth understanding of when image-to-text error occurs.

The average Levenshtein distance in this sample study is 0.23 , which translates to $99.93 \%$ of all characters being, on average, correct. The word recognition is, on average, $99.06 \%$. Hence $99 \%$ of all the 
words in the digitized sample also exist in the manually curated material. The results for each observation appear in Figure $7 B$ and Figure $7 C$.

[Figure 7. about here]

Text-to-Documents Errors. We evaluate the quality of the segmentation using the number of times one text block consists of multiple articles, which indicates that the segmentation of the material has not respected the articles' boundaries. We know there are extensive text-to-documents errors since the OCR engine returns text blocks rather than articles (our documentary unit of choice). In our case the text-todocuments error is less problematic, since we focus mainly on word frequencies. Figure $7 A$ shows how often textual content from different news articles combines into the same text block. Less than one in five (15.97\%) of the studied pages contain any issues with segmentation quality. The notable error in 2019 occurs on a page with crossword puzzles, a feature notoriously difficult to segment but of less relevance for our purposes. Most text-to-documents errors exist in the early data material, a consequence of NLS using different segmentation techniques during different periods, reducing corpus within-corpus comparability. Our text-to-documents error estimate is relatively high, indicating that this complex multi-column corpus can benefit from further efforts to reduce text-to-documents errors.

Document-to-Corpus Error. For our study, we choose to enrich the corpus with three different variables, representing different content types, by training three classification models to predict the content types (Task 1-3) of previously unseen data. We compare the predictive performance of linear and non-linear classifiers to allow for non-linear interactions between input variables. We use (1) logistic regression (LR) as a linear classifier and (2) Random Forest (RF, Breiman 2001) and (3) Extreme Gradient Boosting (XGB, Chen and Guestrin 2016) as non-linear classifiers. Henceforth, we refer to these three classifiers as LR, RF, and XGB. We focus on using mostly visual information-e.g. the position of text blocks on the page and the number of words in the text block-as input features. We believe that not 
using the textual content as input features decreases the correlation between the predicted meta-data labels and the document-to-corpus error, hence trading improved within-corpus comparability for increased document-to-corpus error. We also believe that, even if including textual content as input features may reduce the variance of the documents-to-corpus error, it may also increase the bias. See Appendix A.1 for more details.

Figure 7D shows the F1-score (Sokolova and Lapalme 2009) for the models and the prediction tasks. The F1-score captures a weighted average between how many of the correct text blocks the classifier identifies (precision) and how many of the selected text blocks are correct (recall). A high F1-scoreclose to $1-$ indicates a perfect classification of the annotated material in the test set. Figure $7 D$ shows that the non-linear classifiers outperform the linear classifier in all three tasks. The results also show how XGB and RF perform equally well when predicting editorial content (Task 1 ) and editorial body text (Task 2), while the XGB classifier achieves the highest F1-score when classifying section titles (Task 3). The logistic regression fails at labeling section titles completely. We find that most text blocks misclassified as section titles are, in fact, either "subsection titles" or page headers including dates and the names of newspapers. In the group of text blocks incorrectly classified as lacking section titles, we find observations of low OCR quality, which increases the number of characters in text blocks. The highest F1scores achieved are $\mathbf{0 . 8 1 7 ,}, 0.848$, and 0.682 for the editorial content, editorial body text, and section titles, giving us also an estimate of the magnitude of the document-to-corpus error of $0.183,0.152$, and 0.318 , respectively. ${ }^{15}$ Based on both the estimated documents-to-corpus error and the qualitative inspection, we decide not to use the meta-data of section titles in downstream analysis as we do not find them usable in separating national from international news.

Processing Errors. To create a final, processed, machine-readable corpus, we want to transfer the machine-readable corpus from the original ALTO-format ${ }^{16}$ to a txt-format. This transformation enables us to read the data using standard software, such as Python or R, for our analysis. We use the discrepancies 
between the content of the ALTO files and that of the final txt files for a small sample of files as an estimate of overall processing error. We do not detect any discrepancies between the data in these two formats, and thus conclude the processing error is small. However, when investigating the coverage error (see Figure 8a below) we see a spike in the number of pages from Svenska Dagbladet during the late 1940 s due to duplicate copies in the material. We attribute this to a processing error by the NSL.

Measuring the Research Inference Error. The difficulties in measuring the RIE ${ }^{17}$ stem from the fact that researchers typically have little or no way of knowing the perfect answer to their research questions (if we knew, we would not need to conduct our study in the first place). Furthermore, the research inference errors relate closely to the specific research questions. Therefore, we evaluate the research inference errors in relation to our research question: when and how much immigration has been discussed in the national news in Swedish national media?

Coverage Error. Coverage errors are especially difficult to measure since it is often impossible to know how many documents a given target population contains. In our case, the target population is the national newspaper discourse. For our case study, we focus on studying the four national-focused newspapers with a large circulation today. Hence, the coverage error in our case study consists of (1) texts from newspapers other than the four selected for this study, (2) missing texts from the four largest newspapers, and (3) missing meta-data. While we know that many prominent newspapers existed besides the four included in our study, we cannot access all newspapers printed during our study's seventy-five-year span. Hence, we have an immeasurable potential source of coverage error. We reason, however, that the difference between our four newspapers and the others are probably relatively small. The second type of coverage error is more easily studied. Since our resources do not permit manual inspection of the full corpus, we calculate a rough estimate of the coverage error coming from (2) inspecting the data volume. If the number of texts does not show any obvious signs of missing data, we can speculate that the coverage error probably does not risk bias estimates extracted from the data. 
[Figure 8. about here]

The results in Figure $8 A$ show there are no periods in which the number of texts from any of the newspapers is (close to) zero. The increased coverage error for all newspapers during 2019 reflects our only having had access to the material from the first half of that year. We also observe drops in the numbers of documents in specific periods, e.g. in 1976 and 1993 for Dagens Nyheter. Going back to the digitized material for manual inspection, we do not find any missing newspaper editions. We believe that the drop in the number of text blocks is due to the fact the number of words per text block increases. These combined results indicate that the coverage error regarding available texts from the four largest newspapers is relatively small. ${ }^{18}$

Lastly, our estimates of the documents-to-corpus error indicate that we do not have the meta-data necessary to separate national from international news. As such, we have a substantial meta-datarelated coverage error (3).

Sampling Error. By this time, we have learned that the meta-data-related coverage error is large, since we cannot separate national from international news using meta-data. We try to mitigate this problem by changing our operationalization from the national news to the editorial content of the first part of the papers. The first part of the paper is less likely to contain news content about culture or sports, and hence this sample, we believe, would better generalize our results to national news. We can easily extract the text blocks from the first part of the newspaper by looking at the page number meta-data automatically generated by the OCR procedure. Furthermore, we use the labels produced by the RF classifier to select the text blocks more probable to contain editorial content, since we consider the documents-to-corpus error associated with Task 2 acceptable. At the same time, by using a sample of pages we introduce a sampling error. Here, the sample is also non-random, and hence, we might introduce both bias and variance into our conclusions. 
Text Curation Error. Before measuring the salience of immigration discourse, we curate the raw texts by removing all uncommon words and stop words and by transforming important trigrams and bigrams into unigrams (e.g. "Olof Palme" becomes "olof_palme"). We also remove all non-alphanumerical characters and replace all numbers with a generic token signifying "numbers." Finally, we remove all documents containing fewer than fifteen tokens. ${ }^{19}$ Our curation efforts aim to reduce the vocabulary size, removing uncommon words - many due to image-to-text errors - that risk distorting measures of topic salience proportion. The size of the introduced text curation errors is difficult to quantify meaningfully, for example by measuring the ratio of tokens from the original texts left in the data after the curation processes (e.g. in this case study, since we cannot easily disentangle whether a change in this proportion is due to curation or to structural changes in the source material).

Specification Error. In our case study, we calculate the immigration salience using sets of keywords associated with immigration. We use both a "strict" and a "broad" set of keywords as a sensitivity analysis. The strict version contains the Swedish words for refugee ("flykting"), immigration ("invandring"), and asylum seeker ("asylsökande"), ${ }^{20}$ while the broad keywords allow all inflections on those terms. We are aware that this simple-but commonly used-keyword-based approach is prone to its own specification errors (Eads et al. 2021; King, Lam, and Roberts 2017). We estimate the specification error by comparing how the substantial analysis differs depending on the choice of keywords.

Figure $8 B$ shows the immigration-salience analysis. We can see that the two sets of keywords lead to some slight differences in interpretation. Most notably, there is a clear-and expected - difference in level. The level difference was most pronounced during the European "refugee crisis" of 2015-2016. Using the strict keyword set, we find that immigration salience increased by $151.7 \%$ during $2014-2015$, while the broad keyword set yields an increase of $216.8 \%$. Furthermore, the broad keywords show decreasing salience during 1999-2000, while the strict keywords show an increase. This example shows 
the problems associated with high specification error, namely the difference between the research question we wish to answer and the way we operationalize it for our given material.

\section{Corpus Comparability}

Measuring Within-Corpus Comparability. Figure 7A shows how the text-to-documents error is larger before 1970 than afterward. The changing techniques for segmentation used by the NLS decrease the text-to-documents error for the later period, but also decreases within-corpus comparability. This is one example of a trade-off between different error components, showing how fixing one type of error

may reduce corpus comparability. Figure 9 also shows that the documents-to-corpus error in Task 1 and Task 2 are (relatively) constant over time, while the error for Task 3 fluctuates much more. Furthermore, it seems that the specification error is particularly problematic for 2015 , considered by many a turning point in the Swedish media discourse (Emilsson 2020) (see Figure 8B).

\section{[Figure 9. about here]}

From the combined results we can conclude that this corpus has some issues with within-corpus quality, due partly to changes in the source material and partly to changes in digitization processes. We also note that some of our decisions in the data curation processes may have had negative effects on withincorpus comparability. However, all newspapers show similar trends for most errors, and therefore comparison between newspapers is likely feasible. Furthermore, closer inspections of the material do not suggest that we must avoid cross-time comparison, but does indicate we must take care in conducting diachronic analysis, due to the potential error structures' effect on the conclusion (Nelson 2017).

Measuring Between-Corpus Comparability. If we wish to compare the topic salience found in this example to similar work, for example Eberl et al. (2018), we need to consider the between-corpus comparability of this corpus. We may evaluate between-corpus comparability by comparing the error 
structure of the total corpus error. However, we know that the available unit of analysis (text blocks) is conditioned on the segmentation procedure and differs from the unit perceived by humans reading the material (news article). As such, to make direct comparisons of salience estimates between corpora using different units of analysis may not be meaningful. Furthermore, the error in the classification of editorial body text may also make between-corpus comparisons more difficult if other corpora have better meta-data quality.

\section{Corpus Reproducibility}

The NLS has administered the digitization of the Swedish National News Corpus 1945-2019 for many decades. We have neither access to the exact details of the original images nor the resources necessary to restart the digitization process anew in its entirety. Thus, it is practically difficult to reproduce this corpus from the original analog material. The original images taken from the original newspaper pages are, however, available at an on-site NLS data lab open to researchers. Starting with these images, researchers may attempt to re-extract the textual content. Furthermore, any researcher with access to the on-site lab can freely run our code ${ }^{21}$ to reproduce our corpus.

We also study inter-coder reliability for the task of enriching the corpus with additional meta-data. High values in the measures of inter-coder reliability indicate that the different coders extract the same information from the same page. Low inter-coder reliability indicates the labels are highly subjective, and thus difficult to replicate. In Table 2 we present three measures of inter-coder reliability: Agreement, Cohen's $\kappa$, and F1-score (Artstein and Poesio 2008; Sokolova and Lapalme 2009). Agreement is simply a measure of how often the coders agree, while Cohen's $\kappa$ also incorporates how often the coders might agree just by chance. The F1-score is a weighted measure of the number of false positives and false negatives within the agreement.

[Table 2. about here] 
Table 2 shows that inter-coder reliability is lowest (furthest from 1) for Task 2 . We manually explore where disagreement amongst the annotators occurs. The most disagreement stems from a full-page advertisement, where the content of the page has the same style as traditional editorial content. One of the annotators has missed the small text indicating the text is indeed an advertisement and not journalistic editorial content. An additional common source of disagreement occurs when paragraph headings within an article get separated from the body text following. One of the annotators tends to label such paragraph titles as body text, but the others label them as titles. The manual examination of the disagreement indicates that most errors are avoidable, and thus the randomness in the data curation reducible. We believe that the availability of the code used to create the corpus, and the reasonably high inter-coder reliability, make the reproducibility of the corpus relatively high.

\section{Total Corpus Quality}

Our case study illustrates not only that each corpus has different strengths and weaknesses but also that for each corpus we need to consider multiple dimensions and errors in our analysis. It also shows the need to balance different errors given the resources available. The image-to-text error in the Swedish National Newspaper Corpus 1945-2019 is small and constant over time, yielding processed machinereadable textual content of high quality, also in comparison with other historical newspaper corpora (Pääkkönen et al. 2016; Tanner et al. 2009). We view the size of the documents-to-corpus error (which is also constant over time) for the predicted label identifying editorial body text as acceptable to answer our research question. The different newspapers show similar patterns and trends over time, indicating that the corpus is suitable for cross-paper comparison. However, the results also indicate that we need to work closely with the source material to avoid erroneous conclusions in cross-time studies.

[Table 3. about here] 


\section{Conclusion}

All empirical studies include uncertainty due to potential errors in data. Given that the social sciences have gained a rich experience in using computational text analysis methods for large-scale corpora over recent decades, we suggest that it is now time to turn to a systematic analysis of the role of data quality in scientific inference. It is time to open the door into the messy kitchen.

Creating a multifaceted textual corpus for diverse social inquiry is no quick and easy task. It typically requires time- and cost-intensive manual labor, especially if it includes digitization procedures. Due to limited resources, researchers must consider both which errors they wish to reduce and which errors they can afford to reduce. This paper has introduced the total corpus quality framework to aid in the systematic analysis that can aid these decisions. By breaking down total corpus quality into its components, it suggests how future research can identify where the smallest investments may produce the biggest impacts, yielding better scientific results from textual data.

As reflected in our discussions of the total corpus quality framework and the presented exemplary case study, some quality dimensions are easily measurable and technically alleviated. Textual representation errors are more technical in nature and most often independent of specific research questions. We suggest that institutions, such as libraries and archives, hold great potential to assist in the corpus creation process, mitigate textual representation errors, and help to store large corpora of interest for many social science researchers. Other components of the total corpus error dimension are more difficult to reduce and require decisions by researchers with different questions.

We view the total corpus quality framework as a tool for researchers to make such decisions: to take different potential aspects of data quality into account at all stages of data curation and before using large-scale computational text analysis. All types of data can be erroneous, but "what is important is to know the type and form in order to be able to gauge its effects" (Franzosi 2004:172). We do not believe it essential to produce a numerical estimate for all dimensions and components of the total corpus 
quality framework. Instead, one should view it as a tool for evaluating the strengths and limitations of a corpus to answer a specific research question, as well as to identify different areas with the potential to improve the quality of the corpus in relation to the research question. Researchers can utilize the introduced error types and quality dimensions, as well as their effects on bias and variance, the better to evaluate and to discuss the reliability and validity of scientific insights based on specific corpora. As such, we still face unresolved challenges in creating best practices and easy-to-use rules of thumb for how best to estimate and correct the many types of errors that weaken the fitness of use of textual data for social scientific research questions which future work must continue developing. 


\section{References}

Agiatis, Benardou, Erik Champion, Costis Dallas, and Lorna M. Hughes. 2017. "Introduction: A Critique of Digital Practices and Research Infrastructures." Pp. 1-14 in Cultural Heritage Infrastructures in Digital Humanities, edited by B. Agiatis, E. Champion, C. Dallas, and L. M. Hughes. London, UK: Routledge.

Amaya, Ashley, Paul P. Biemer, and David Kinyon. 2020. "Total Error in a Big Data World: Adapting the TSE Framework to Big Data." Journal of Survey Statistics and Methodology 8:89-119.

Artstein, Ron and Massimo Poesio. 2008. "Inter-Coder Agreement for Computational Linguistics." Computational Linguistics 34:555-96.

Baevski, Alexei., Henry Zhou, Abdelrahman Mohamed, and Michael Auli. 2020. "Wav2vec 2.0: A Framework for Self-Supervised Learning of Speech Representations." Pp. 12449-60 in Advances in Neural Information Processing Systems 33. https://proceedings.neurips.cc/paper/2020/file/92d1e1eb1cd6f9fba3227870bb6d7f07-Paper.pdf.

Bail, Christopher A. 2012. "The Fringe Effect: Civil Society Organizations and the Evolution of Media Discourse about Islam since the September 11th Attacks." American Sociological Review 77:855-79.

Bail, Christopher A. 2016a. "Combining Natural Language Processing and Network Analysis to Examine How Advocacy Organizations Stimulate Conversation on Social Media." Proceedings of the National Academy of Sciences 113:11823-28.

Bail, Christopher A. 2016b. "Cultural Carrying Capacity: Organ Donation Advocacy, Discursive Framing, and Social Media Engagement." Social Science \& Medicine 165:280-88.

Bais, Frank, Barry Schouten, Peter Lugtig, Vera Toepoel, Judit Arends-Tòth, Salima Douhou, Natalia 
Kieruj, Mattijn Morren, and Corrie Vis. 2019. “Can Survey Item Characteristics Relevant to Measurement Error Be Coded Reliably? A Case Study on 11 Dutch General Population Surveys." Sociological Methods \& Research 48:263-95.

Barberá, Pablo, Amber. E. Boydstun, Suzanna Linn, Ryan McMahon, and Jonathan Nagler. 2021. "Automated Text Classification of News Articles: A Practical Guide." Political Analysis 29:19-42.

Baumer, Eric P. S., David Mimno, Shion Guha, Emily Quan, and Geri K. Gay. 2017. “Comparing Grounded Theory and Topic Modeling: Extreme Divergence or Unlikely Convergence?" Journal of the Association for Information Science and Technology 68:1397-1410.

Belur, Jyoti, Lisa Tompson, Amy Thornton, and Miranda Simon. 2021. "Interrater Reliability in Systematic Review Methodology: Exploring Variation in Coder Decision-Making." Sociological Methods \& Research 50:837-65.

Biemer, Paul P. 2010. "Total Survey Error: Design, Implementation, and Evaluation." Public Opinion Quarterly 74:817-48.

Biemer, Paul P. and Lars E. Lyberg. 2003. Introduction to Survey Quality. Hoboken, NJ: John Wiley \& Sons.

Björk, Lars. 2015. How Reproductive Is a Reproduction? Digital Transmission of Text Based Documents. Borås, Sweden: University of Borås.

Bode, Katherine. 2020. “Why You Can't Model Away Bias.” Modern Language Quarterly 81:95-124.

Boyd, Danah and Kate Crawford. 2012. "Critical Questions for Big Data: Provocations for a Cultural, Technological, and Scholarly Phenomenon." Information, Communication \& Society 15:662-79.

Breiman, Leo. 2001. “Random Forests." Machine Learning 45:5-32.

Broman, Karl, Mine Cetinkaya-Rundel, Amy Nussbaum, Christopher Paciorek, Roger Peng, Daniel Turek, 
and Hadley Wickham. 2017. "Recommendations to Funding Agencies for Supporting Reproducible Research." American Statistical Association. Retrieved September 17, 2021

(https://www.amstat.org/asa/files/pdfs/pol-reproducibleresearchrecommendations.pdf).

Bruns, Axel and Tim Highfield. 2013. "Political Networks on Twitter: Tweeting the Queensland State Election." Information, Communication \& Society 16:667-91.

Camerer, Colin F., Anna Dreber, Eskil Forsell, Teck-Hua Ho, Jürgen Huber, Magnus Johannesson, Michael Kirchler, Johan Almenberg, Adam Altmejd, Taizan Chan, Emma Heikensten, Felix Holzmeister, Taisuke Imai, Sir Isaksson, Gideon Nave, Thomas Pfeiffer, Michael Razen, and Hang Wu. 2016. “Evaluating Replicability of Laboratory Experiments in Economics.” Science 351:1433-36.

Campbell, John L., Charles Quincy, Jordan Osserman, and Ove K. Pedersen. 2013. “Coding In-Depth Semistructured Interviews: Problems of Unitization and Intercoder Reliability and Agreement." Sociological Methods \& Research 42:294-320.

Chaudhuri, Arijit and Horst Stenger. 2005. Survey Sampling: Theory and Methods. Boca Raton, FL: CRC Press.

Chen, Tianqi and Carlos Guestrin. 2016. "Xgboost: A Scalable Tree Boosting System." Pp. 785-94 in Proceedings of the 22nd ACM SIGKDD International Conference on Knowledge Discovery and Data Mining. https://doi.org/10.1145/2939672.2939785.

Cochran, William G. 1977. Sampling Techniques. 3rd ed. New York, NY: John Wiley \& Sons.

Conway, Paul. 2011. "Archival Quality and Long-Term Preservation: A Research Framework for Validating the Usefulness of Digital Surrogates." Archival Science 11:293-309.

Dannélls, Dana, Torsten Johansson, and Lars Björk. 2019. “Evaluation and Refinement of an Enhanced OCR Process for Mass Digitisation." Pp. 124-32 in Digital Humanities in the Nordic Countries. 
http://ceur-ws.org/Vol-2364/9_paper.pdf.

Demarest, Leila and Arnim Langer. 2019. “How Events Enter (or Not) Data Sets: The Pitfalls and Guidelines of Using Newspapers in the Study of Conflict." Sociological Methods \& Research. Retrieved September 21, 2021 (https://doi.org/10.1177/0049124119882453).

Denny, Matthew J. and Arthur Spirling. 2018. "Text Preprocessing for Unsupervised Learning: Why It Matters, When It Misleads, and What to Do about It." Political Analysis 26:168-89.

DiMaggio, Paul, Manish Nag, and David Blei. 2013. “Exploiting Affinities between Topic Modeling and the Sociological Perspective on Culture: Application to Newspaper Coverage of U.S. Government Arts Funding." Poetics 41:570-606.

Dobson, Miriam and Benjamin Ziemann. 2020. Reading Primary Sources: The Interpretation of Texts from Nineteenth and Twentieth Century History. 2nd ed. London, UK: Routledge.

Downing, Steven M. 2004. "Reliability: On the Reproducibility of Assessment Data." Medical Education 38:1006-12.

Eads, Alicia, Alexandra Schofield, Fauna Mahootian, David Mimno, and Rens Wilderom. 2021. "Separating the Wheat from the Chaff: A Topic and Keyword-Based Procedure for Identifying Research-Relevant Text*约." Poetics 86. https://doi.org/10.1016/j.poetic.2020.101527.

Eberl, Jakob-Moritz, Christine E. Meltzer, Tobias Heidenreich, Beatrice Herrero, Nora Theorin, Fabienne Lind, Rosa Berganza, Hajo G. Boomgaarden, Christian Schemer, and Jesper Strömbäck. 2018. “The European Media Discourse on Immigration and Its Effects: A Literature Review." Annals of the International Communication Association 42(3):207-23.

Edelmann, Achim, Tom Wolff, Danielle Montagne, and Christopher A. Bail. 2020. “Computational Social Science and Sociology." Annual Review of Sociology 46:61-81. 
Emilsson, Henrik. 2020. "Continuity or Change? The Impact of the Refugee Crisis on Swedish Political Parties' Migration Policy Preferences." Pp. 99-121 in Forced Migration and Resilience, edited by M. Fingerle and R. Wink. Wiesbaden, Germany: Springer.

European Statistical System Committee. 2011. "European Statistics Code of Practice." Publications Office of the European Union. Retrieved July 15, 2021 (https://ec.europa.eu/eurostat/web/quality/european-statistics-code-of-practice).

Evans, James A. and Pedro Aceves. 2016. "Machine Translation : Mining Text for Social Theory." Annual Review of Sociology 42:21-50.

Fickers, Andreas. 2012. "Towards A New Digital Historicism?" Journal of European History and Culture 1:1-9.

Fligstein, Neil, Jonah Stuart Brundage, and Michael Schultz. 2017. "Seeing Like the Fed: Culture, Cognition, and Framing in the Failure to Anticipate the Financial Crisis of 2008." American Sociological Review 82:879-909.

Flores, René D. 2017. “Do Anti-Immigrant Laws Shape Public Sentiment? A Study of Arizona's SB 1070 Using Twitter Data." American Journal of Sociology 123:333-84.

Franzosi, Roberto. 1989. “From Words to Numbers: A Generalized and Linguistics-Based Coding Procedure for Collecting Textual Data." Sociological Methodology 19:263-98.

Franzosi, Roberto. 2004. From Words to Numbers: Narrative, Data, and Social Science. Cambridge, UK: Cambridge University Press.

Freese, Jeremy and David Peterson. 2017. "Replication in Social Science." Annual Review of Sociology 43:147-65.

Fuhse, Jan, Oscar Stuhler, Jan Riebling, and John Levi Martin. 2020. "Relating Social and Symbolic 
Relations in Quantitative Text Analysis. A Study of Parliamentary Discourse in the Weimar Republic." Poetics 78. https://doi.org/10.1016/j.poetic.2019.04.004.

Garcia, David and Bernard Rimé. 2019. "Collective Emotions and Social Resilience in the Digital Traces After a Terrorist Attack." Psychological Science 30:617-28.

Garg, Nikhil, Londa Schiebinger, Dan Jurafsky, and James Zou. 2018. “Word Embeddings Quantify 100 Years of Gender and Ethnic Stereotypes." Proceedings of the National Academy of Sciences 115:E3635-44.

Geiger, R. Stuart, Kevin Yu, Yanlai Yang, Mindy Dai, Jie Qiu, Rebekah Tang, and Jenny Huang. 2020. “Garbage in, Garbage out? Do Machine Learning Application Papers in Social Computing Report Where Human-Labeled Training Data Comes From?" Pp. 325-36 in Proceedings of the 2020 Conference on Fairness, Accountability, and Transparency. https://doi.org/10.1145/3351095.3372862.

Gentzkow, Matthew, Bryan Kelly, and Matt Taddy. 2019. "Text as Data." Journal of Economic Literature $57: 535-74$.

Giglietto, Fabio, Luca Rossi, and Davide Bennato. 2012. "The Open Laboratory: Limits and Possibilities of Using Facebook, Twitter, and YouTube as a Research Data Source." Journal of Technology in Human Services 30:145-59.

Golafshani, Nahid. 2003. "Understanding Reliability and Validity in Qualitative Research." The Qualitative Report 8:597-607.

Grimmer, Justin. 2010. “A Bayesian Hierarchical Topic Model for Political Texts : Measuring Expressed Agendas in Senate Press Releases." Political Analysis 18(1):1-35.

Grimmer, Justin, Margaret E. Roberts, and Brandon M. Stewart. 2021. “Machine Learning for Social 
Science: An Agnostic Approach." Annual Review of Political Science 24:395-419.

Grimmer, Justin and Brandon M. Stewart. 2013. "Text as Data : The Promise and Pitfalls of Automatic Content Analysis Methods for Political Texts." Political Analysis 21:267-29.

Harris, Verne. 2002. "The Archival Sliver: Power, Memory, and Archives in South Africa." Archival Science 2:63-86.

Hawkins, Douglas M. 2004. "The Problem of Overfitting." Journal of Chemical Information and Computer Sciences 44:1-12.

Hill, Mark J. and Simon Hengchen. 2019. "Quantifying the Impact of Dirty OCR on Historical Text Analysis: Eighteenth Century Collections Online as a Case Study." Digital Scholarship in the Humanities 34:825-43.

Hoffman, Mark Anthony, Jean-Philippe Cointet, Philipp Brandt, Newton Key, and Peter Bearman. 2018. "The (Protestant) Bible, the (Printed) Sermon, and the Word(s): The Semantic Structure of the Conformist and Dissenting Bible, 1660-1780." Poetics 68:89-103.

Hsieh, Yuli Patrick and Joe Murphy. 2017. "Total Twitter Error." Pp. 23-46 in Total survey error in practice, edited by P. P. Biemer, E. de Leeuw, S. Eckman, B. Edwards, F. Kreuter, L. E. Lyberg, C. N. Tucker, and B. T. West. Hoboken, NJ: John Wiley \& Sons.

Hurtado Bodell, Miriam, Martin Arvidsson, and Måns Magnusson. 2019. “Interpretable Word Embeddings via Informative Priors." Pp. 6324-30 in Proceedings of the 2019 Conference on Empirical Methods in Natural Language Processing and the 9th International Joint Conference on Natural Language Processing (EMNLP-IJCNLP). https://doi.org/10.18653/v1/D19-1661.

Iraci, Joe, Richard Hess, and Kimberley Flak. 2020. "The Digitization of Audiotapes." Canadian Conservation Institute. Retrieved July 21, 2021 (https://www.canada.ca/en/conservation- 
institute/services/conservation-preservation-publications/technical-bulletins/digitization-audiotapes.html).

Jarlbrink, Johan and Pelle Snickars. 2017. "Cultural Heritage as Digital Noise: Nineteenth Century Newspapers in the Digital Archive." Journal of Documentation 73:1228-43.

Jo, Eun Seo and Timnit Gebru. 2020. "Lessons from Archives: Strategies for Collecting Sociocultural Data in Machine Learning." Pp. 306-16 in Proceedings of the 2020 Conference on Fairness, Accountability, and Transparency. https://doi.org/10.1145/3351095.3372829.

Juran, Joseph M. and Joseph A. De Feo. 2010. Juran's Quality Handbook: The Complete Guide to Performance Excellence. New York, NY: McGraw-Hill Education.

King, Gary, Patrick Lam, and Margaret E. Roberts. 2017. “Computer-Assisted Keyword and Document Set Discovery from Unstructured Text." American Journal of Political Science 61:971-88.

Kozlowski, Austin C., Matt Taddy, and James A. Evans. 2019. "The Geometry of Culture: Analyzing the Meanings of Class through Word Embeddings." American Sociological Review 84:905-49.

Liu, David M. and Matthew J. Salganik. 2019. "Successes and Struggles with Computational Reproducibility: Lessons from the Fragile Families Challenge." Socius 5:1-21.

Lohr, Sharon L. 2019. Sampling: Design and Analysis. 2nd ed. Boca Raton, FL: Chapman and Hall/CRC. Lyberg, Lars. 2012. "Survey Quality." Survey Methodology 38:107-30.

Manovich, Lev. 2016. "The Science of Culture? Social Computing, Digital Humanities and Cultural Analytics." Journal of Cultural Analytics 1. https://doi.org/10.22148/16.004.

Mellon, Jonathan and Christopher Prosser. 2017. "Twitter and Facebook Are Not Representative of the General Population: Political Attitudes and Demographics of British Social Media Users." Research 
\& Politics 4:1-9. https://doi.org/10.1177/2053168017720008.

Mesiti, Marco, Roberto Celle, Matteo A. Sorrenti, and Giovanna Guerrini. 2006. "X-Evolution: A System for XMLschema Evolution and Document Adaptation." Pp. 1143-46 in International Conference on Extending Database Technology. https://doi.org/10.1007/11687238_78.

Mitchell, Ryan. 2018. Web Scraping with Python: Collecting More Data from the Modern Web. Sebastopol, CA: O’Reilly Media, Inc.

Mogalakwe, Monageng. 2009. “The Documentary Research Method--Using Documentary Sources in Social Research." Eastern Africa Social Science Research Review 25:43-58.

Mohr, John W. 1998. "Measuring Meaning Structures." Annual Review of Sociology 24:345-70.

Mohr, John W., Christopher A. Bail, Margaret Frye, Jennifer C. Lena, Omar Lizardo, Terence E. McDonnell, Ann Mische, Iddo Tavory, and Frederick F. Wherry. 2020. Measuring Culture. New York, NY: Columbia University Press.

Molina, Mario and Filiz Garip. 2019. "Machine Learning for Sociology." Annual Review of Sociology 45:27-45.

Murphy, Kevin P. 2012. Machine Learning: A Probabilistic Perspective. Cambridge, MA: MIT Press.

Mützel, Sophie. 2015. "Structures of the Tasted: Restaurant Reviews in Berlin Between 1995 and 2012." Pp. 147-67 in Moments of Valuation: Exploring Sites of Dissonance, edited by A. B. Antal, M. Hutter, and D. Stark. Oxford, UK: Oxford University Press.

Navarro, Gonzalo. 2001. "A Guided Tour to Approximate String Matching." ACM Computing Surveys (CSUR) 33:31-88.

Nelson, Laura K. 2017. “Computational Grounded Theory: A Methodological Framework.” Sociological 
Methods \& Research 49:3-42.

Nelson, Laura K. 2019. “To Measure Meaning in Big Data, Don't Give Me a Map, Give Me Transparency and Reproducibility." Sociological Methodology 49:139-43.

Nguyen, Dong, Maria Liakata, Simon DeDeo, Jacob Eisenstein, David Mimno, Rebekah Tromble, and Jane Winters. 2020. "How We Do Things with Words: Analyzing Text as Social and Cultural Data." Frontiers in Artificial Intelligence 3. https://doi.org/10.3389/frai.2020.00062.

Olsen, W. 2004. "Triangulation in Social Research: Qualitative and Quantitative Methods Can Really Be Mixed." Developments in Sociology 20:103-18.

Olteanu, Alexandra, Carlos Castillo, Fernando Diaz, and Emre Kiciman. 2019. "Social Data: Biases, Methodological Pitfalls, and Ethical Boundaries." Frontiers in Big Data 2. https://doi.org/10.3389/fdata.2019.00013.

Open Science Collaboration. 2015. "Estimating the Reproducibility of Psychological Science." Science 349:943-53.

Pääkkönen, Tuula, Jukka Kervinen, Asko Nivala, Kimmo Kettunen, and Eetu Mäkelä. 2016. “Exporting Finnish Digitized Historical Newspaper Contents for Offline Use." D-Lib Magazine 22. Retrieved September 17, 2021 (https://dlib.org/dlib/july16/paakkonen/07paakkonen.print.html).

Pechenick, Eitan Adam, Christopher M. Danforth, and Peter Sheridan Dodds. 2015. “Characterizing the Google Books Corpus: Strong Limits to Inferences of Socio-Cultural and Linguistic Evolution." PloS One 10. https://doi.org/10.1371/journal.pone.0137041.

Pipino, Leo L., Yang W. Lee, and Richard Y. Wang. 2002. “Data Quality Assessment." Communications of the ACM 45:211-18.

Read, Robert Lee. 2000. “Mapping Defects or Dirt Dynamically Affecting an Image Acquisition Device." 
U.S. Patent 6,035,072, issued March 7, 2000.

https://patentimages.storage.googleapis.com/72/ef/a6/18e356a814cbc9/US6035072.pdf

Roberts, Margaret E., Brandon M. Stewart, and Dustin Tingley. 2019. "Stm: An R Package for Structural Topic Models." Journal of Statistical Software 91:1-40.

Rule, Alix, Jean-Philippe Cointet, and Peter S. Bearman. 2015. “Lexical Shifts, Substantive Changes, and Continuity in State of the Union Discourse, 1790-2014." Proceedings of the National Academy of Sciences 112:10837-44.

Ruths, Derek and Jürgen Pfeffer. 2014. "Social Media for Large Studies of Behavior." Science 346:106364.

Salganik, Matthew J. 2017. Bit by Bit: Social Research in the Digital Age. Princeton, NJ: Princeton University Press.

Schofield, A. and D. Mimno. 2016. “Comparing Apples to Apple: The Effects of Stemmers on Topic Models." Transactions of the Association for Computational Linguistics 4:287-300.

Schofield, Alexandra, Måns Magnusson, and David Mimno. 2017. "Pulling out the Stops: Rethinking Stopword Removal for Topic Models." Pp. 432-36 in Proceedings of the 15th Conference of the European Chapter of the Association for Computational Linguistics: Volume 2, Short Papers. https://aclanthology.org/E17-2069.pdf.

Scott, John. 1990. A Matter of Record: Documentary Sources in Social Research. Cambridge, UK: Polity Press.

Sen, Indira, Fabian Flöck, Katrin Weller, Bernd Weiss, and Claudia Wagner. 2021. "A Total Error Framework for Digital Traces of Human Behavior on Online Platforms." Public Opinion Quarterly 85. https://doi.org/10.1093/poq/nfab018. 
Shinde, Archana A. and D. G. Chougule. 2012. "Text Pre-Processing and Text Segmentation for OCR." International Journal of Computer Science Engineering and Technology 2:810-12.

Smith, Ray. 2007. "An Overview of the Tesseract OCR Engine." Pp. 629-33 in Ninth international conference on document analysis and recognition (ICDAR 2007). https://doi.org/10.1109/ICDAR.2007.437699.

Sokolova, Marina and Guy Lapalme. 2009. "A Systematic Analysis of Performance Measures for Classification Tasks." Information Processing \& Management 45:427-37.

van Strien, Daniel, Kaspar Beelen, Mariona Coll Ardanuy, Kasra Hosseini, Barbara McGillivray, and Giovanni Colavizza. 2020. "Assessing the Impact of OCR Quality on Downstream NLP Tasks." Pp. 484-96 in Proceedings of the 12th International Conference on Agents and Artificial Intelligence. https://doi.org/10.17863/CAM.52068.

Tahmasebi, Nina and Simon Hengchen. 2019. "The Strengths and Pitfalls of Large-Scale Text Mining for Literary Studies." Samlaren: Tidskrift För Svensk Litteraturvetenskaplig Forskning 140:198-227.

Tanner, Simon, Trevor Muñoz, and Pich Hemy Ros. 2009. “Measuring Mass Text Digitization Quality and Usefulness." D-Lib Magazine 15. Retrieved September 17, 2021 (https://www.dlib.org/dlib/july09/munoz/07munoz.html).

Terras, Melissa. 2011. “Artefacts and Errors: Acknowledging Issues of Representation in the Digital Imaging of Ancient Texts." Pp. 43-61 in Kodikologie und Paläographie im digitalen Zeitalter 2/Codicology and Palaeography in the Digital Age 2, edited by F. Fischer, C. Fritze, and G. Vogeler. Norderstedt, Germany: Books on Demand.

The Library of Congress. 2016. “ALTO: Technical Metadata for Layout and Text Objects." The Library of Congress. Retrieved September 14, 2021 (https://www.loc.gov/standards/alto/). 
United Nations. 2014. "Fundamental Principles of National Official Statistics." United Nations. Retrieved September 15, 2021 (https://unstats.un.org/unsd/dnss/gp/fundprinciples.aspx).

Wagner-Pacifici, Robin. 2010. "Theorizing the Restlessness of Events." American Journal of Sociology 115:1351-86.

Wang, William Yang. 2017. “'Liar, Liar Pants on Fire': A New Benchmark Dataset for Fake News Detection." Pp. 422-26 in Proceedings of the 55th Annual Meeting of the Association for Computational Linguistics (Volume 2: Short Papers). https://doi.org/10.18653/v1/P17-2067.

Wilkerson, John and Andreu Casas. 2017. "Large-Scale Computerized Text Analysis in Political Science: Opportunities and Challenges." Annual Review of Political Science 20:529-44.

Zhan, Yaowen, Weiqiang Wang, and Wen Gao. 2006. “A Robust Split-and-Merge Text Segmentation Approach for Images." Pp. 1002-5 in 18th International Conference on Pattern Recognition (ICPR'06). Vol. 2. https://doi.org/10.1109/ICPR.2006.169. 


\section{Figures}

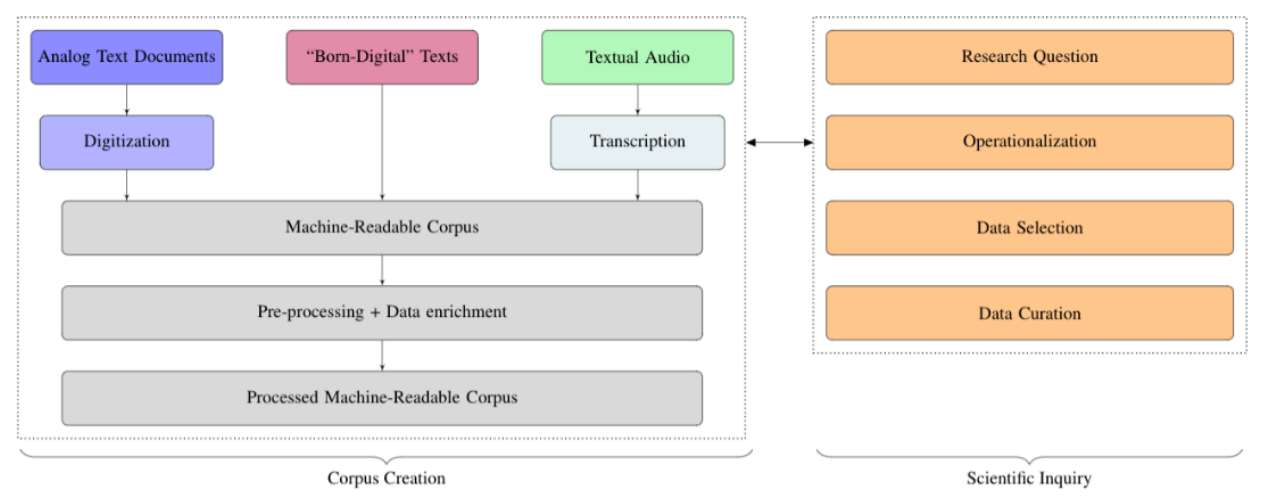

Figure 1.Using text as data in scientific applications

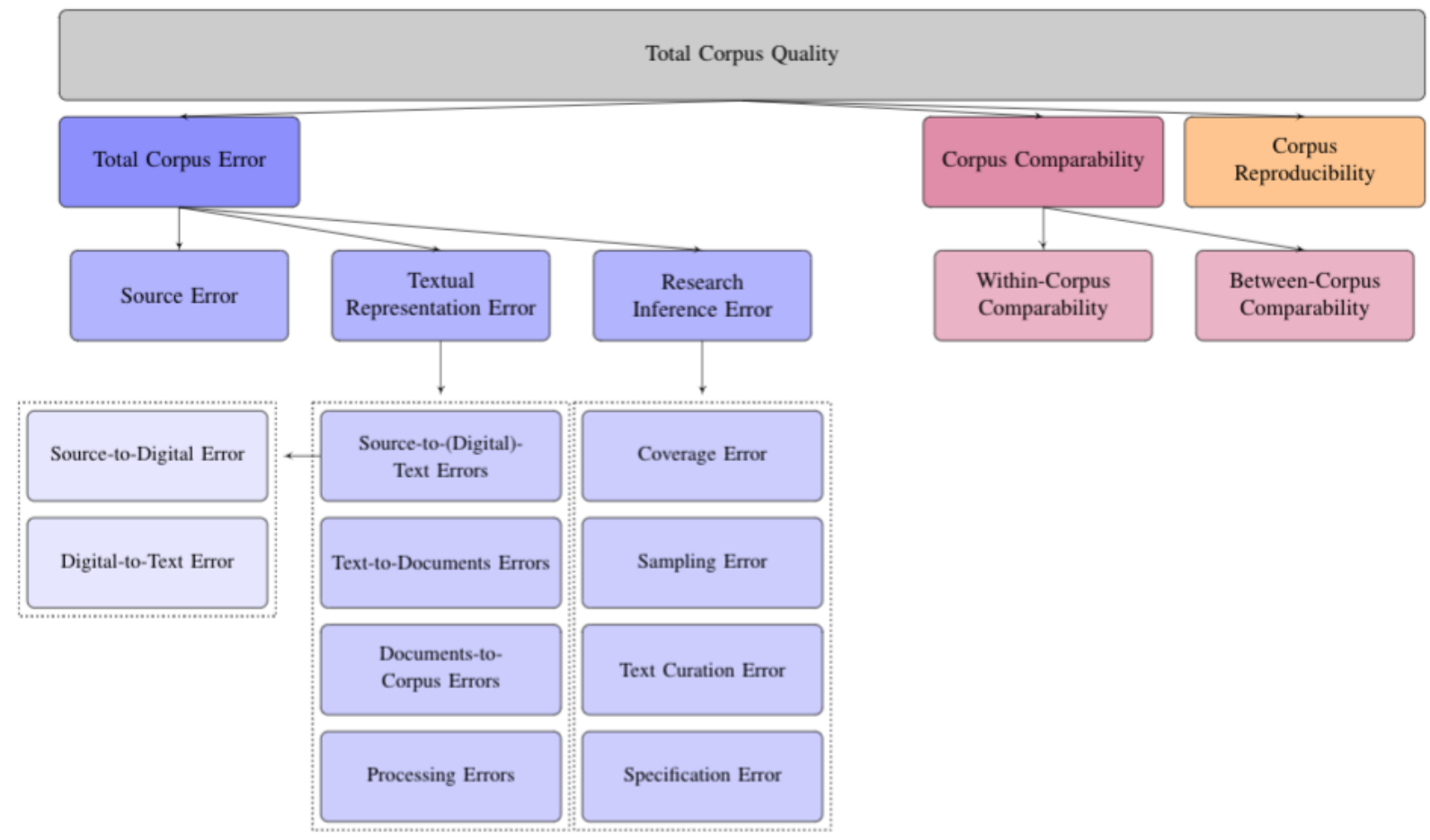

Figure 2. Schematic representation of dimensions and their elements within the total corpus quality framework 


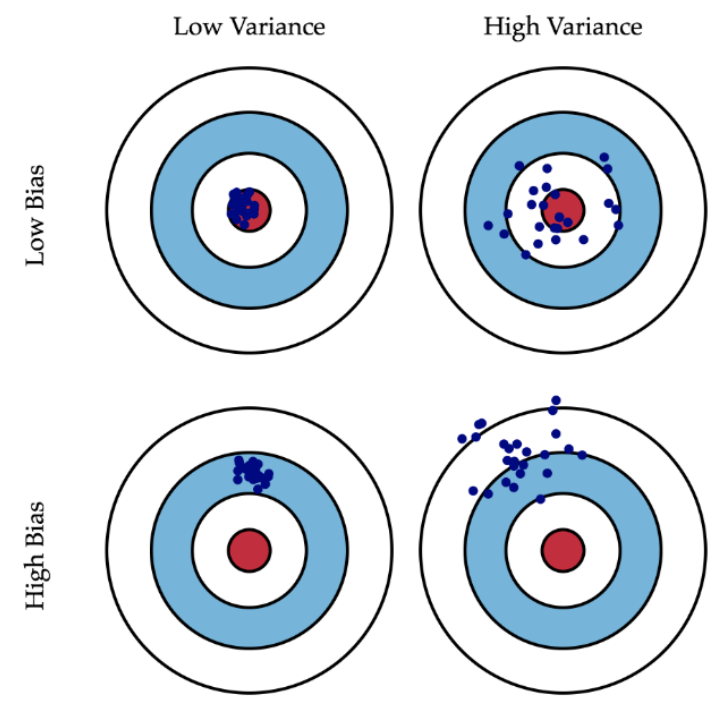

Figure 3. Graphical representation of bias and variance, source: http://scott.fortmann-roe.com/docs/BiasVariance.html

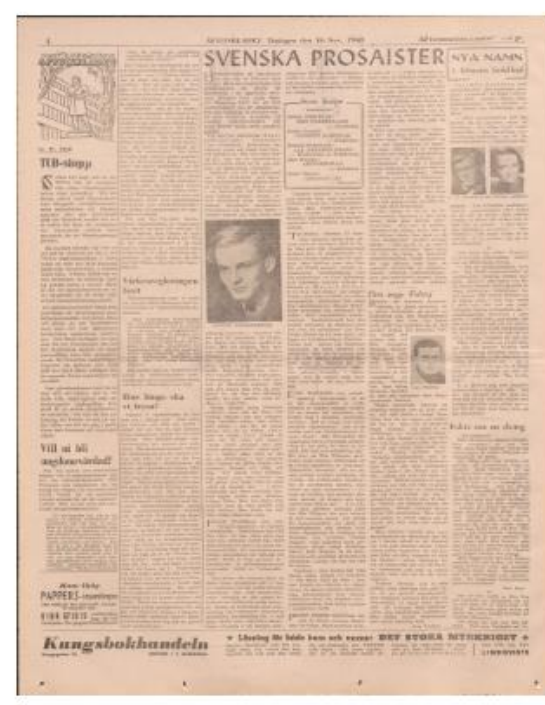

(a)

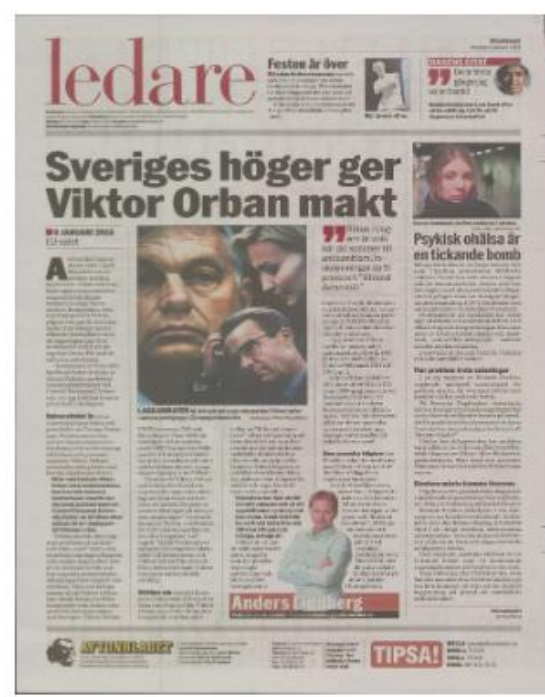

(b)

Figure 4. (a) Editorial page in Aftonbladet, 1945 (b) Editorial page in Aftonbladet, 2019 


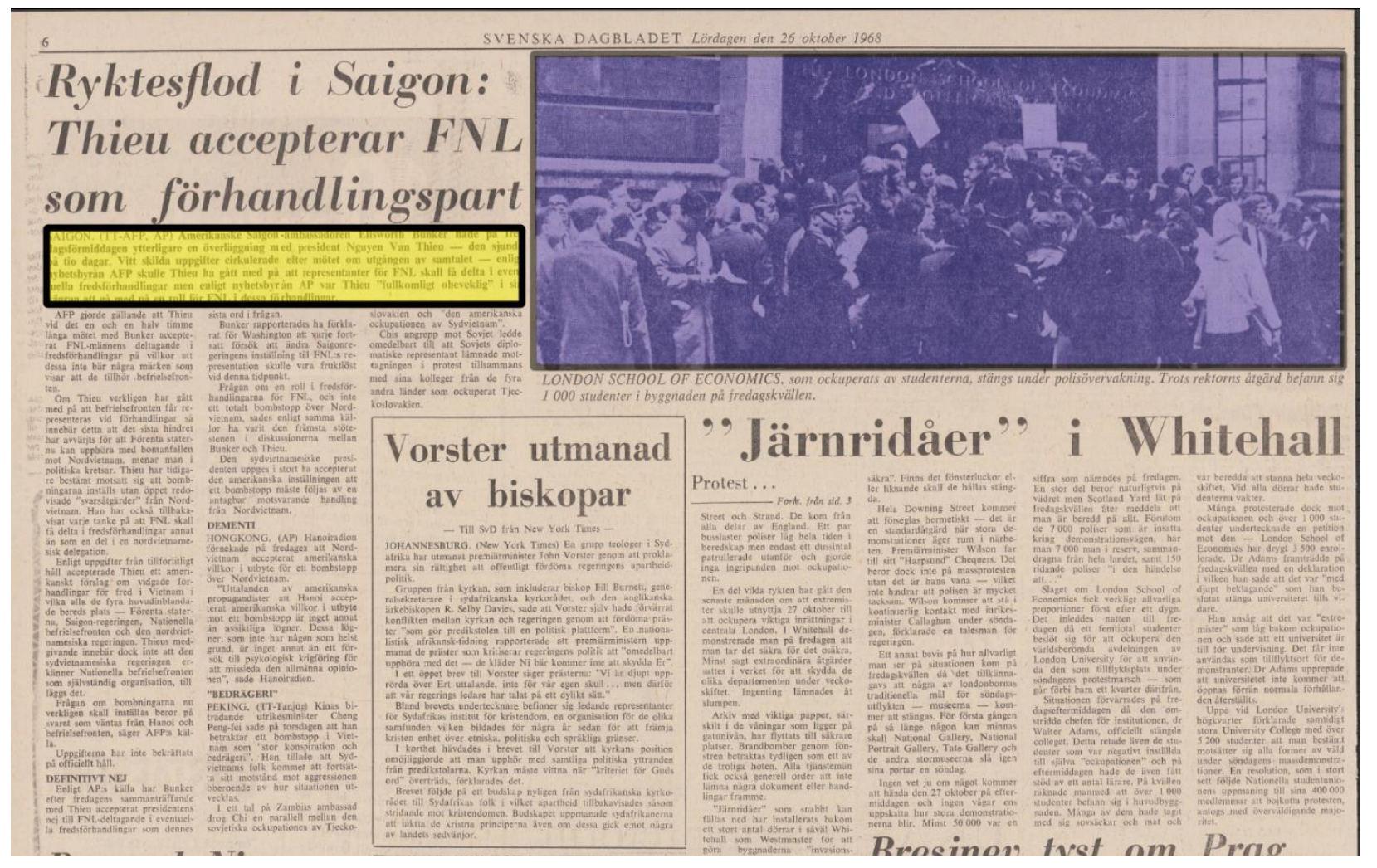

Figure 5. Yellow highlight shows what the segmentation procedure has identified as a text block. In this example the text block corresponds to the lead of the article. Blue highlights what the digitalization procedure has identified as a picture. 


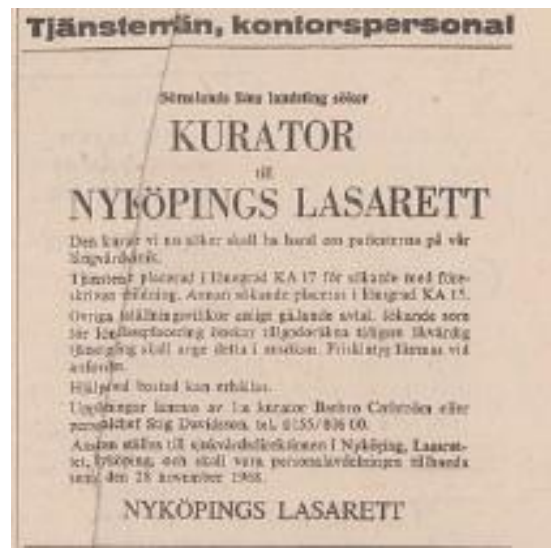

Figure 6. Example of a source-to-digital error. Page is folded, which warps the textual content, effectively making the " $K$ " in NYKÖPING unreadable.
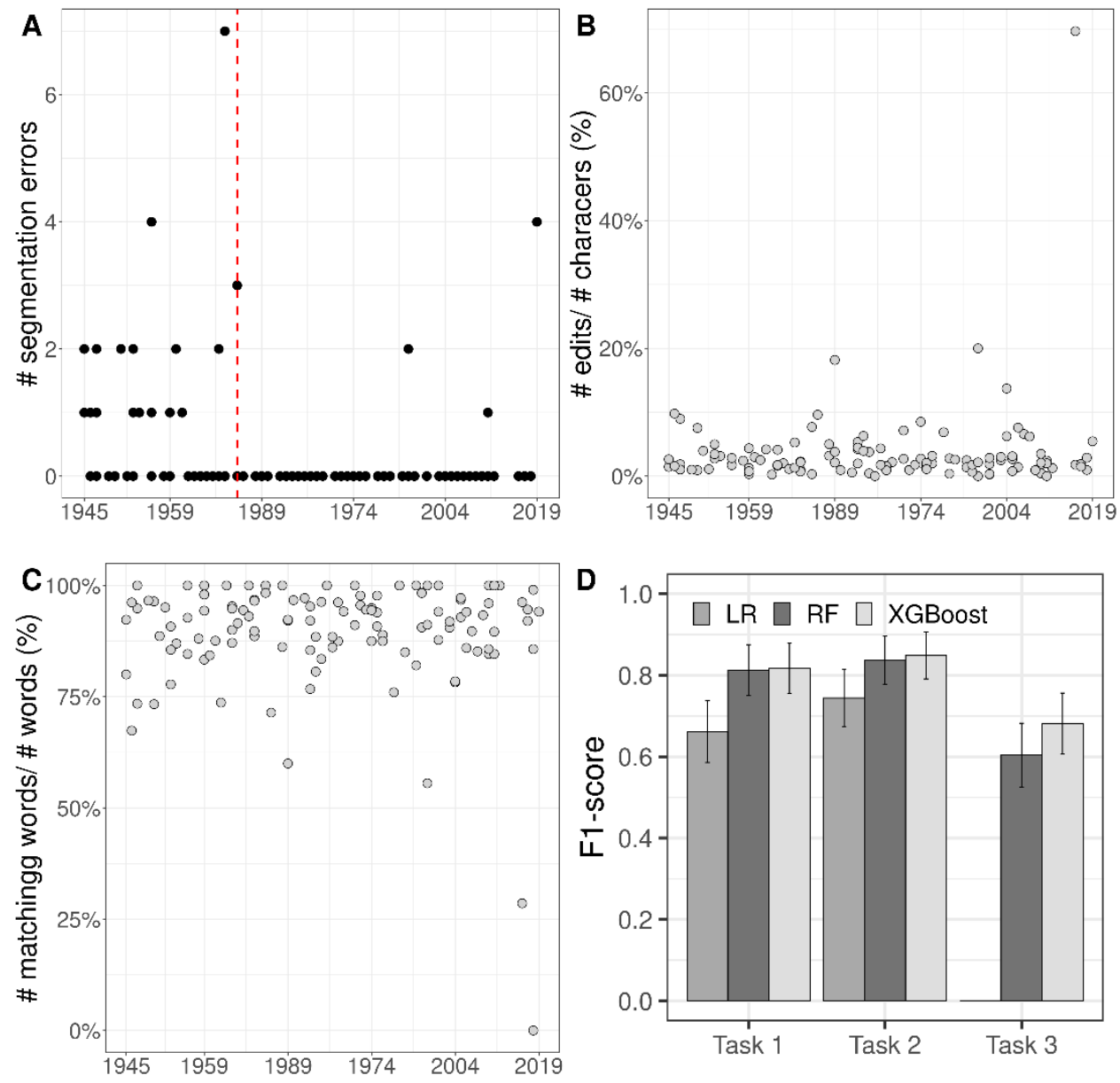

Figure 7. (A) Text-to-documents errors-per-year. The $Y$-axis represents the number of times the segmentation algorithm mixes texts from different articles in a text block. The dashed line represents 1970. (B) Image-to-text error using Levenshtein distance per year. The number of edits needed to turn machine-readable text to gold standard text divided by the total number of characters. Black points represent the Levenshtein distance on the raw material; grey points represent the Levenshtein distance after basic pre-processing. (C) Image-to-text error using word recognition per year. The proportion of words in the machinereadable text that exist in the gold standard. Black points represent the Levenshtein distance on the raw material; grey points represent the Levenshtein distance after basic pre-processing. (D) Documents-to-corpus error. F1-score for the three 
classification tasks in the case study using the three different models: logistic regression (LR), Random Forest (RF), and XGBoost (XGB). Task 1 is to predict editorial content, Task 2 is to predict editorial body text, and Task 3 is to predict whether a text is a section title. Shade represents the model. Error bands calculated using normal approximation by treating every page as independent.

A

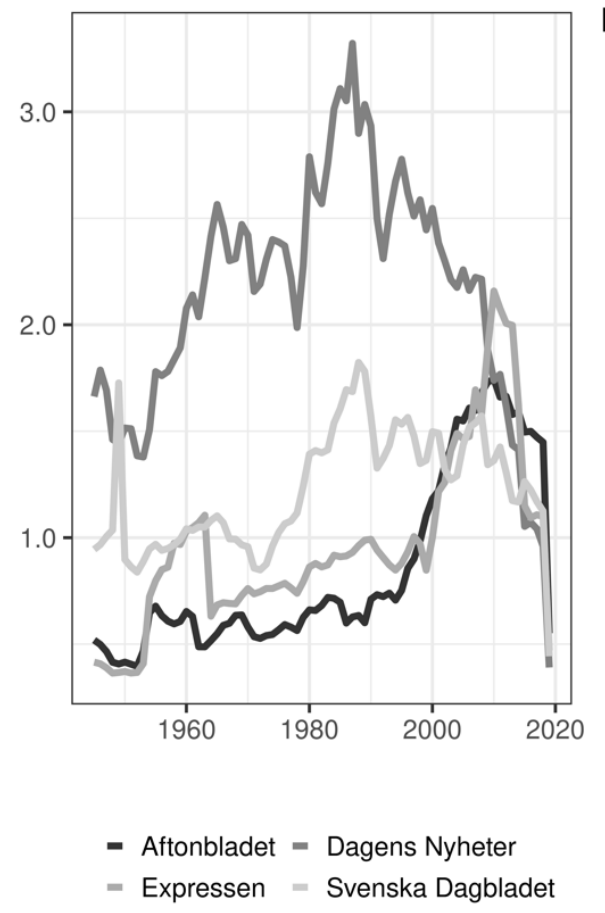

B

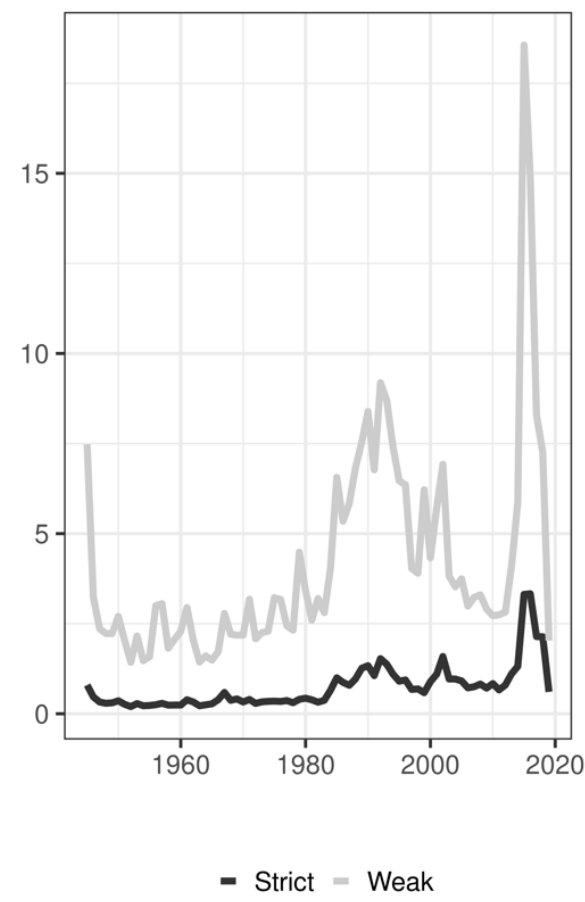

Figure 8. (A) Coverage error. The number of text blocks per year in Sweden's four largest national newspapers in the unprocessed raw source material (in millions). (B) Specification error. The number of occurrences (in thousands) of keywords using "strict" (black) and "weak" (grey) lists of keywords. 


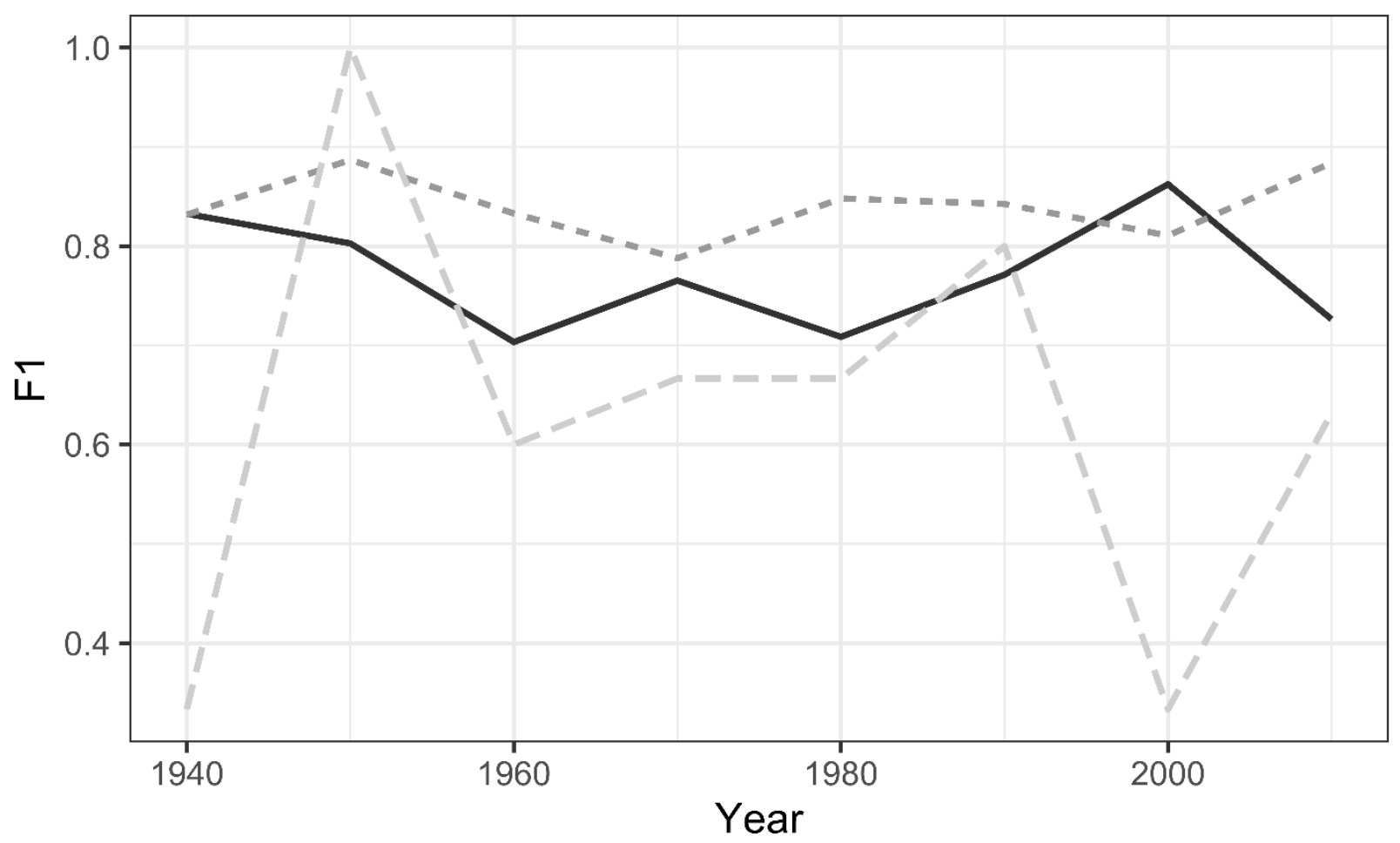

- Task 1 - Task 2 - Task 3

Figure 9. F1-score of XGBoost classifier on hold-out data per decade and task. 


\section{Tables}

\begin{tabular}{llllc}
\hline Error Component & Error Sub-Component & Error type & Bias & Variance \\
\hline Source Error & & & Low & High \\
\hline & Source-to-(Digital)-Text Errors & Source-to-Digital Errors & High & High \\
& Digital-to-Text Errors & High & Low \\
Textual Representation Error & Text-to-Documents Errors & & High & Low \\
& Documents-to-Corpus Errors & & High & High \\
& Processing Error & & High & High \\
\hline & & Coverage Error & High & Low \\
Research Inference Error & & Sampling Error & High & High \\
& & Text Curation Error & High & Low \\
& & Specification Error & High & Low \\
\hline
\end{tabular}

Table 1. Summary of general risk of contributing to bias or variance by error component

\begin{tabular}{llll}
\hline Classification task & Agreement & Cohen's $\kappa$ & F1 \\
\hline Task 1 & 0.963 & 0.926 & 0.960 \\
Task 2 & 0.955 & 0.802 & 0.826 \\
Task 3 & 0.997 & 0.881 & 0.883 \\
\hline
\end{tabular}

Table 2. Inter-coder reliability

\begin{tabular}{|c|c|c|c|c|}
\hline Dimension & Compenent & Sub-Component & Error type & Quality Evaluation \\
\hline \multirow{12}{*}{ Total Corpus Error } & Source Error & & & Low \\
\hline & & & Source-to-Digital Error & High \\
\hline & & Source-to-(Digital)-Text Errors & Digital-to-Text Error & Mcdium \\
\hline & Textual Representation Error & Text-to-Documents Errors & & Low \\
\hline & & & Task 1 & Medium \\
\hline & & Documents-to-Corpus Errors & Task 2 & Mcdium \\
\hline & & & Task 3 & Low \\
\hline & & Processing Errors & & High \\
\hline & & & Coverage Eirror & High \\
\hline & Research Inference Error & & Sampling Ërror & High \\
\hline & & & Text-Curation Error & High \\
\hline & & & Specification Error & Low \\
\hline \multirow{2}{*}{ Corpus Comparability } & & Within-Corpus Comparability & & Mcdium \\
\hline & & Between-Corpus Comparahility & & \\
\hline Corpus Reproducibility & & & & Mcdium \\
\hline
\end{tabular}

Table 3. Summary of the quality (from low to high) with regards to a specific error when used in our case study using keyword search in the Swedish National Newspaper Corpus 1945-2019 to answer the research question: when and how much has the national news in Swedish national media discussed immigration? Quality evaluation represents our combined evaluation per quality dimension. 



\section{Footnotes}

${ }^{1}$ We use the term 'data curation' for all efforts in data management to maintain and increase data's usability.

${ }^{2}$ Moreover, agencies involved in the production of statistical surveys have further defined dimensions and components to assess data quality (e.g. the fundamental principles of national official statistics for the United Nations 2014 or the European Statistical System Committee 2011).

${ }^{3}$ When we henceforth use the terms 'original source material' or 'source material,' we mean this observed source material including source errors, and not the true, error-free, source material.

${ }^{4}$ Here we use the term 'original material' to refer to the source material including any potential source errors.

${ }^{5}$ This process is not necessary for digital-born data, as the source material is already digital.

${ }^{6}$ If one uses random sampling, measuring sampling errors can be possible.

7 If we are interested only in saying something about the actual documents, then coverage error and frame error would coincide. We choose to use the concept of coverage error due to the vagueness of the target population as regards textual data (as discussed previously). Coverage error also relates to the non-response error of the total survey quality framework in that, if text collection was specifically for research purposes, we would consider nonresponse error to be part of the coverage error.

8 If the corpus contains different editions identical in content, or duplicates, the sampling error would still be zero after their removal.

${ }^{9}$ Replicability is a related concept describing the extent to which one may repeat a study without using the original data but generally using the same methods for similar results (Broman et al. 2017; Freese and Peterson 2017). We do not use this term since it does not apply to corpus creation.

${ }^{10} \mathrm{NLS}$ uses the archive digitization software Zissor, of which ABBYY is the OCR engine. The segmentation procedure uses "segmentation rules," different sets of rules to identify cohesive texts within each newspaper each decade. Segmentation rules also update when the layout of a newspaper changes in a significant way (see Dannélls, Johansson, and Björk 2019 for more details).

${ }^{11}$ We use a stratified random sample with replacement and unequal selection probabilities, stratified by runs of five years (e.g. 1945-1949, 1950-1955) for each newspaper (Cochran 1977; Lohr 2019). In total this will make up the number of five-year groups times the number of newspapers $(15 \times 4)$ yielding sixty strata. In each stratum, the sample size is two to enable estimation of the variance within each stratum. If more than one edition is available for a sampled issue, we randomly select one of them. The cost of this simplicity is a slight inflation of the variance in the sample. We address the inflated variances by presenting results using the Hansen-Hurwitz estimator (Chaudhuri and Stenger 2005).

${ }^{12}$ From each of the sampled 120 pages we sample one "paragraph" within which to evaluate the image-to-text error. The annotator goes through each page and gives an ID to each paragraph identified on that page. We define a paragraph as a recognizable cohesive block of text not separated by blank lines. We would thus treat multiple paragraphs separated by only an indention as one paragraph. Titles and subtitles we treat as separate paragraphs if they do not share the same line with the following text. We treat image captions as paragraphs if they include more information than the name of the person in the picture or photograph. Short notices and citations with larger fonts-but not tables or infographics - we also include as paragraphs. When recording all the paragraphs, we sample one paragraph ID randomly using random sampling. We use special characters to recreate and highlight specific layouts (e.g. squares used to highlight the beginning of a new paragraph).

${ }^{13}$ Again, we use stratified random sampling to create a gold standard. For the meta-data, to create a training data set we use one stratum per year and newspapers of size 2 (year multiplied by number of newspaper times size $=$ $75 \times 4 \times 2=600$ pages). However, for the test data we again use five-year periods (number of five-year periods times the number of newspaper times size $=15 \times 4 \times 3=120$ pages). Combined, this results in 51,133 text blocks in the training data and 10,002 text blocks in the test data set.

${ }^{14}$ Both annotators provide labels for 30 of 600 pages, containing 1,543 text blocks in total.

${ }^{15}$ We get the errors by calculating $(1-F 1)$. If the predictions correspond perfectly with the gold standard $(F 1=$ 1 ), the meta-data error is non-existing.

${ }^{16}$ ALTO is an XML-schema used to store different physical text resources, such as e-books and newspapers, and includes information regarding layout and font information (The Library of Congress 2016). 
${ }^{17}$ The sampling error is, however, easy to measure if we employ random sampling.

${ }^{18}$ However, the coverage error in terms of missing texts from other papers may still be substantial.

${ }^{19}$ We curate the raw texts by removing all words which occur fewer than twenty-five times in each of the four different newspapers or which occur fewer than fifty times in total. We also remove all words which appear more than five million times, i.e. mostly stop words and common image-to-text errors. We further curate the corpus by combining the tokens making up the names of all prime ministers, ministers of finance, and ministers in charge of immigration policy and immigration policy. We also connect common and (potentially) immigration-related bigrams and trigrams. We replace all digits 0-9 with the token "NN." Due to the copyright protection of the source material, we remove all documents with fewer than fifteen tokens, a criterion imposed by the National Swedish Library. ${ }^{20}$ Clearly, this is a simplified example with many potential keywords missing. However, we believe it suffices as an illustrative example. Furthermore, one can evaluate the size of the specification error testing robustness across different operationalizations. However, extensive comparison between keyword search and alternative approaches is beyond the scope of this case study.

${ }^{21}$ https://github.com/ A link to a Github repository with code for reproduction of data will appear here upon acceptance. Excluded for anonymity reasons at present. 